ARTICLE

DOI: $10.1038 / \mathrm{s} 41467-018-05924-9$

\title{
TBC1d24-ephrinB2 interaction regulates contact inhibition of locomotion in neural crest cell migration
}

Jaeho Yoon ${ }^{1,2}$, Yoo-Seok Hwang ${ }^{1}$, Moonsup Lee ${ }^{1}$, Jian Sun ${ }^{1}$, Hee Jun Cho ${ }^{1,3}$, Laura Knapik ${ }^{1}$ \& Ira O. Daar (1) ${ }^{1}$

Although Eph-ephrin signalling has been implicated in the migration of cranial neural crest (CNC) cells, it is still unclear how ephrinB transduces signals regulating this event. We provide evidence that TBC1d24, a putative Rab35-GTPase activating protein (Rab35 GAP), complexes with ephrinB2 via the scaffold Dishevelled (Dsh) and mediates a signal affecting contact inhibition of locomotion (CIL) in CNC cells. Moreover, we found that, in migrating $\mathrm{CNC}$, the interaction between ephrinB2 and $\mathrm{TBC} 1 \mathrm{~d} 24$ negatively regulates E-cadherin recycling in these cells via Rab35. Upon engagement of the cognate Eph receptor, ephrinB2 is tyrosine phosphorylated, which disrupts the ephrinB2/Dsh/TBC1d24 complex. The dissolution of this complex leads to increasing E-cadherin levels at the plasma membrane, resulting in loss of CIL and disrupted CNC migration. Our results indicate that TBC1d24 is a critical player in ephrinB2 control of CNC cell migration via CIL.

\footnotetext{
${ }^{1}$ National Cancer Institute, Frederick, MD 21702, USA. ${ }^{2}$ Department of Biochemistry, Institute of Cell Differentiation and Aging, College of Medicine, Hallym University, ChunCheon 200702 Kangwon-Do, Republic of Korea. ${ }^{3}$ Immunotherapy Convergence Research Center, Korea Research Institute of Bioscience and Biotechnology (KRIBB), Daejeon 34141, Korea. Correspondence and requests for materials should be addressed to I.O.D. (email: daari@mail.nih.gov)
} 
C ranial neural crest (CNC) cells arise from neuroectoderm in the early neurula embryo and they undergo collective cell migration after segregation from the ectoderm through at least a partial epithelial-to-mesenchymal transition ${ }^{1}$. Various factors are known to participate in neural crest cell migration. Separation from ectoderm involves a coordinated alteration in the levels of E-cadherin and $\mathrm{N}$-cadherin, as well as cadherin-1 $11^{1-9}$. The chemotactic response between $\mathrm{CNC}$ and placodes that secrete attractant molecules such as stromal cellderived factor 1 (SDF-1) affects the migratory direction ${ }^{4}$. Other secreted factors, including C3a, semaphorins, glial-derived growth factor, fibroblast growth factors (FGFs) and vascular endothelial growth factors, also play a role ${ }^{10-17}$. Directionality of CNC cell migration also relies upon the non-canonical Wnt/planar cell polarity (PCP) signalling pathway ${ }^{18,19}$, which even influences mechanical cues from the underlying mesoderm tissue to regulate CNC migration ${ }^{20}$. Additionally, CNC cell migration is critically dependent on cell-to-cell interactions. Recently, several groups have shown that E-cadherin levels are downregulated to initiate $\mathrm{CNC}$ migration, but a low level of E-cadherin is maintained for migrating CNC cells to regulate cell-to-cell adhesion and motility ${ }^{1,3,21}$.

Eph/ephrin signalling is involved in a number of embryonic developmental processes by regulating cell-cell interaction events. Several studies using the mouse, chick and Xenopus systems demonstrate that CNC cells express various combinations of ephrin ligands and Eph receptors to guide directional migration. Loss-of-function studies targeting Eph-ephrin signalling demonstrate that complementary expression of ephrin ligands and Eph receptors generates bi-directional signalling to modulate repulsion or attraction of migratory $\mathrm{CNC}$ cells $^{22-30}$. However, it is still unclear how ephrinB mechanistically transduces the signals affecting this repulsion or attraction.

Here we provide evidence that $\mathrm{TBC} 1 \mathrm{~d} 24$ interacts with ephrinB2. TBC1d24 is a Rab-GAP that has two conserved domains consisting of a TBC (Tre2-Bub2-Cdc16) domain and TLD (TBC LysM) domain, which are predicted to regulate endocytosis and exocytosis of cellular vesicles ${ }^{31}$. In human patients, several mutations in TBC1d 24 have been identified, and heterozygous missense mutations have been determined to cause neurological disorders, including DOORS (deafness, onychodystrophy, osteodystrophy, mental retardation and seizures) and familial infantile myoclonic epilepsy ${ }^{32,33}$. In addition, patients with homozygous $\mathrm{TBC} 1 \mathrm{~d} 24$ truncation mutations display severe neurodegeneration ${ }^{34}$. In our study, loss of TBC1d24 function causes CNC cell migration defects through disruption of CIL, and these defects can be rescued by re-expressing the wild-type protein. However, TBC1d24 interaction mutants that are not able to associate with either ephrinB2 or Rab35 fail to rescue the TBC1d24 loss-of-function phenotype. We show that the ephrinB2 and TBC1d24 interaction modulates contact inhibition of locomotion (CIL) through regulating E-cadherin recycling. Our results provide the molecular mechanism of how ephrinB2 regulates the CIL response during CNC cell migration.

\section{Results}

The newly identified ephrinB2-binding partner, TBC1d24. Several ephrinB-interacting proteins have been discovered that function in pathways regulating cell adhesion and migration (RGS3-PDZ, FGF receptor (FGFR), Dishevelled, Grb4 and CNK1 $)^{35-37}$. To identify additional proteins that might mediate ephrinB signalling, we used mass spectrometric analysis of proteins that co-immunoprecipitate (Co-IP) with ephrinB2 when it is overexpressed in Xenopus embryos ${ }^{38}$. From this analysis, we identified TBC1d24 as a candidate ephrinB2-interacting protein.
Confirmation of a possible interaction between these proteins was provided by Co-IP analysis of lysates from Xenopus embryos exogenously expressing tagged versions of TBC1d24 and ephrinB2 (Fig. 1a). Of the three transmembrane ephrinB ligands (B1, B2, B3), ephrinB2 has the most robust interaction with TBC1d24 in Co-IP analyses (Fig. 1a). IP analysis of ephrinB2 from lysates of LS174T human colon carcinoma cells showed that TBC1d24 was found in the ephrinB2 immune-complexes (Supplementary Fig. 1a), indicating that an endogenous interaction exists between ephrinB2 and TBC1d24. Having established that ephrinB2 can associate with TBC1d24, we expressed TBC1d24 in the embryos along with ephrinB2 deletion mutants and found that deletion of six amino acids in the C-terminus encompassing the PDZ-binding motif markedly reduced the interaction with TBC1d24 (Fig. 1b).

In order to find the region within $\mathrm{TBC} 1 \mathrm{~d} 24$ necessary for an association with ephrinB2, deletion mutants within TBC1d24 were constructed. IP analysis with deletion mutants generated within TBC1d24 (Supplementary Fig. 1b) indicated that the TBC domain was critical for the association between ephrinB2 and TBC1d24 (Supplementary Fig. 1b). Using serial deletion mutants within the TBC domain, it was determined that the 35 amino acids at the $3^{\prime}$ end of the TBC domain (termed the $\mathrm{F}$ region) is required for the interaction (Fig. 1c). To address whether the ephrinB2/TBC1d24 interaction affects their localisation, tagged versions of both proteins were expressed alone or along with either a wild-type or interaction mutant version of each protein. In animal pole explants (ectoderm), TBC1d24 alone localised to the cytoplasm; however, ephrinB2 co-expression promotes membrane localisation of TBC1d24. In contrast, TBC1d 24 remained in the cytoplasm when co-expressed with the ephrinB2 mutant that lacks the PDZ-binding motif (Fig. 1d). A complementary experiment expressing a TBC1d24 mutant lacking the $\mathrm{F}$ region (TBC1d24- $\triangle \mathrm{F}$ ) necessary for an association with ephrinB2 (Fig. 1d) revealed that the TBC1d24- $\Delta \mathrm{F}$ mutant protein remained in the cytoplasm when co-expressed with the ephrinB2 (Fig. 1d). These data indicate that the association between ephrinB2 and TBC1d24 causes the re-localisation of TBC1d24 from the cytoplasm to the plasma membrane and confirm that the interaction is dependent upon the ephrinB2 PDZ-binding motif and 35 amino acids within the TBC domain of $\mathrm{TBC} 1 \mathrm{~d} 24$. The question remained whether the ephrinB2/ TBC1d24 association is direct or mediated through another partner protein.

Dsh mediates the interaction between ephrinB2 and TBC1d24. Dishevelled (Dsh) is a key molecule in Wnt signalling and is also known to be a major player in the Wnt/PCP pathway required for proper neural crest cell migration ${ }^{18,39}$. Previous studies demonstrated that Dsh interacts with the C-terminal PDZ-binding motif of ephrinB ligands ${ }^{40-43}$, which is also the motif required for the interaction with TBC1d24 (Fig. 1d). One possibility is that the ephrinB2-TBC1d24 interaction is mediated through an association with Dsh2. Co-IP analysis was performed with lysates from the embryos exogenously co-expressing tagged versions of TBC1d24 and Dsh2. The results clearly indicate the presence of Dsh2 in TBC1d24 immune-complexes and that of TBC1d24 in Dsh2 immune-complexes (Fig. 2a). In an IP analysis of Dsh2 from lysates of LS174T human colon carcinoma cells, TBC1d24 was detected in the Dsh2 immune-complexes (Supplementary Fig. 1c), indicating that the endogenous proteins are found associated. Co-IP analysis of Dsh2 domain-specific deletion constructs that were co-expressed along with TBC1d24 in the embryos indicates that the formation of a complex between Dsh2 and TBC1d24 was disrupted by deletion of the DEP domain in 


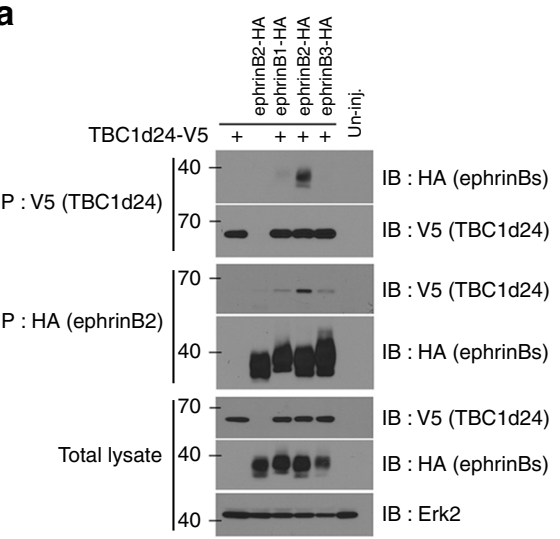

c

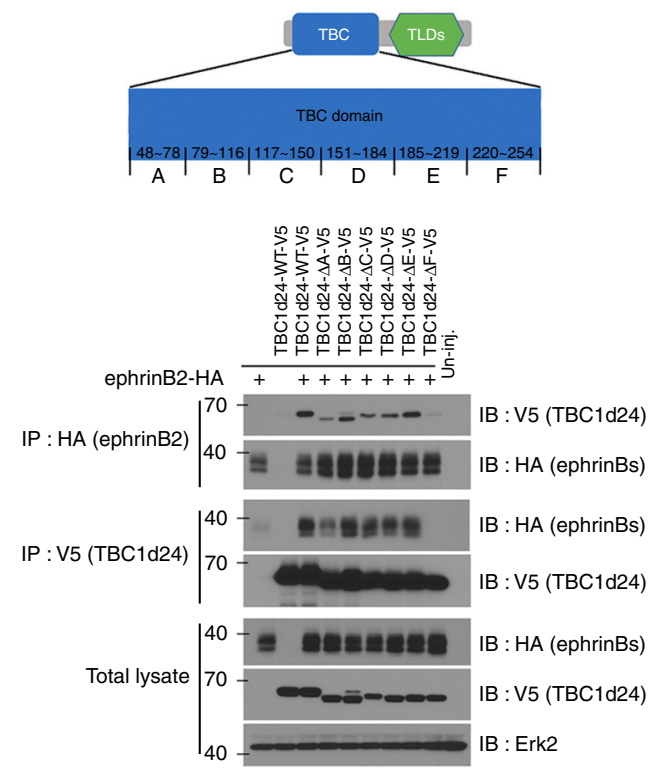

b

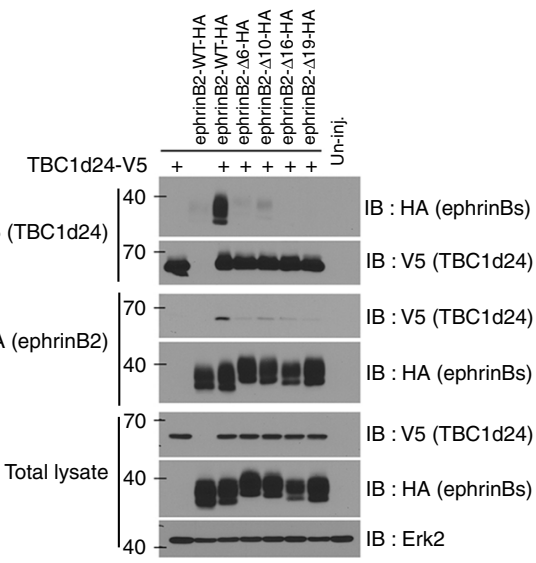

d
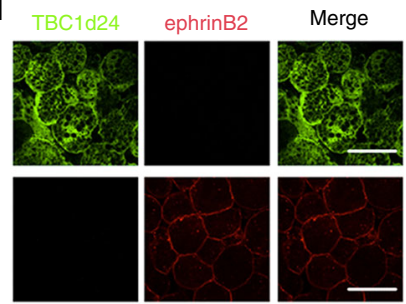

TBC1d24-WT-V5
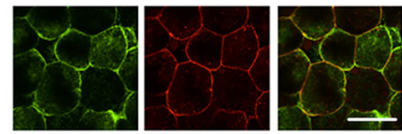

ephrinB2-WT-HA alone
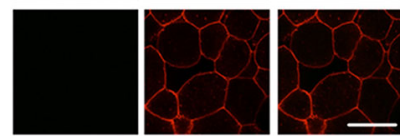

TBC1d24-WT-V5

+ ephrinB2-WT
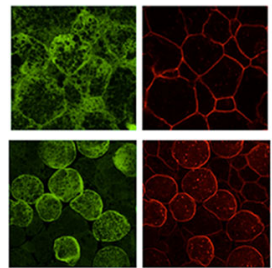

ephrinB2- $\triangle 4-\mathrm{HA}$ alone

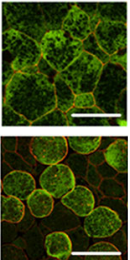

TBC1d24-WT-V5

+ ephrinB2- $44-\mathrm{HA}$

TBC1d24- $\triangle F-V 5$

+ ephrinB2-WT-HA

Fig. 1 TBC1d24 interacts with ephrinB2. a Co-immunoprecipitation assay (Co-IP) using gastrula embryos (stage 10) injected with TBC1d24-V5 RNA (500 pg) and ephrinB1-HA (300 pg), ephrinB2-HA (500 pg) or ephrinB3-HA RNAs (300 pg) show that TBC1d24 interacts with ephrinB2. b Illustration of serial deletion mutants from the C-terminus of ephrinB2. Co-IP using gastrula embryos injected with RNA encoding TBC1d24-V5 and HA-tagged ephrinB2 serial deletion mutants shows that the PDZ binding motif $(\Delta 6)$ of ephrinB2 is required for TBC1d24 interaction. $\mathbf{c}$ Illustration of the amino acid stretches representing regions deleted within the TBC domain. Co-IP using gastrulas injected with RNA for ephrinB2-HA and TBC1d24-V5 serial deletion mutants shows that the $\mathrm{F}$ region within the TBC domain of TBC1d24 is critical for an ephrinB2 interaction. $\mathbf{d}$ Immunofluorescence microscopic analysis (IF) shows that membrane localisation of V5-tagged TBC1d24 was induced by wild-type ephrinB2 but not ephrinB2- $\Delta 4$. Animal caps were dissected at stage 10 and then immunostained for ephrinB2 (red) and TBC1d24 (green). Bar, $50 \mu \mathrm{m}$

Dsh2 (Fig. 2b). A construct representing only the DEP domain associated with TBC1d24 (Fig. 2b). Together, the evidence indicates that the DEP domain is required for an interaction between Dsh2 and TBC1d24. Complementary Co-IP experiments were performed using TBC1d24 domain-specific deletion mutants (Supplementary 1d), as well as specific deletion mutants within the TBC domain (Fig. 2c). The results show that the $\mathrm{F}$ region within the $\mathrm{TBC}$ domain of $\mathrm{TBC} 1 \mathrm{~d} 24$ is required for the interaction with Dsh2 (Fig. 2c).

These IP results were consistent with the possibility that Dsh2 mediates the ephrinB2-TBC1d24 interaction, and in support of this hypothesis is the report showing that the DEP domain of Dsh2 is critical for an association with ephrinB1 ${ }^{42}$. To test this concept, we employed Dsh2 anti-sense morpholino oligonucleotides (Dsh2-MO) to knockdown endogenous Dsh2 in the embryos. Dsh2 morphant embryos displayed a remarkable reduction in the ephrinB2-TBC1d24 interaction, which was recovered by exogenously re-expressing Dsh2 through injecting a MO-resistant Dsh2 RNA (Fig. 2d). As a further test of the requirement for Dsh2, we also examined the localisation of TBC1d24 in embryonic ectoderm in the Dsh2-MO injected embryos (Fig. 2e). Consistent with the Co-IP results, the Dsh2MO reduced TBC1d24 membrane localisation, whereas Dsh2 RNA injection rescued the $\mathrm{TBC} 1 \mathrm{~d} 24$ localisation to the membrane in ephrinB2 co-expressing embryos (Fig. 2e). Taken together, our results show that Dsh2 mediates the ephrinB2-TBC1d24 association.

Phosphorylation of ephrinB2 regulates the interaction with TBC1d24. EphrinB1 can be tyrosine phosphorylated in response to binding the extracellular domain of a cognate Eph receptor $^{44,45}$, the tight-junction-associated protein Claudin ${ }^{46}$ or in response to FGFR activation ${ }^{47}$. These phosphorylation events 


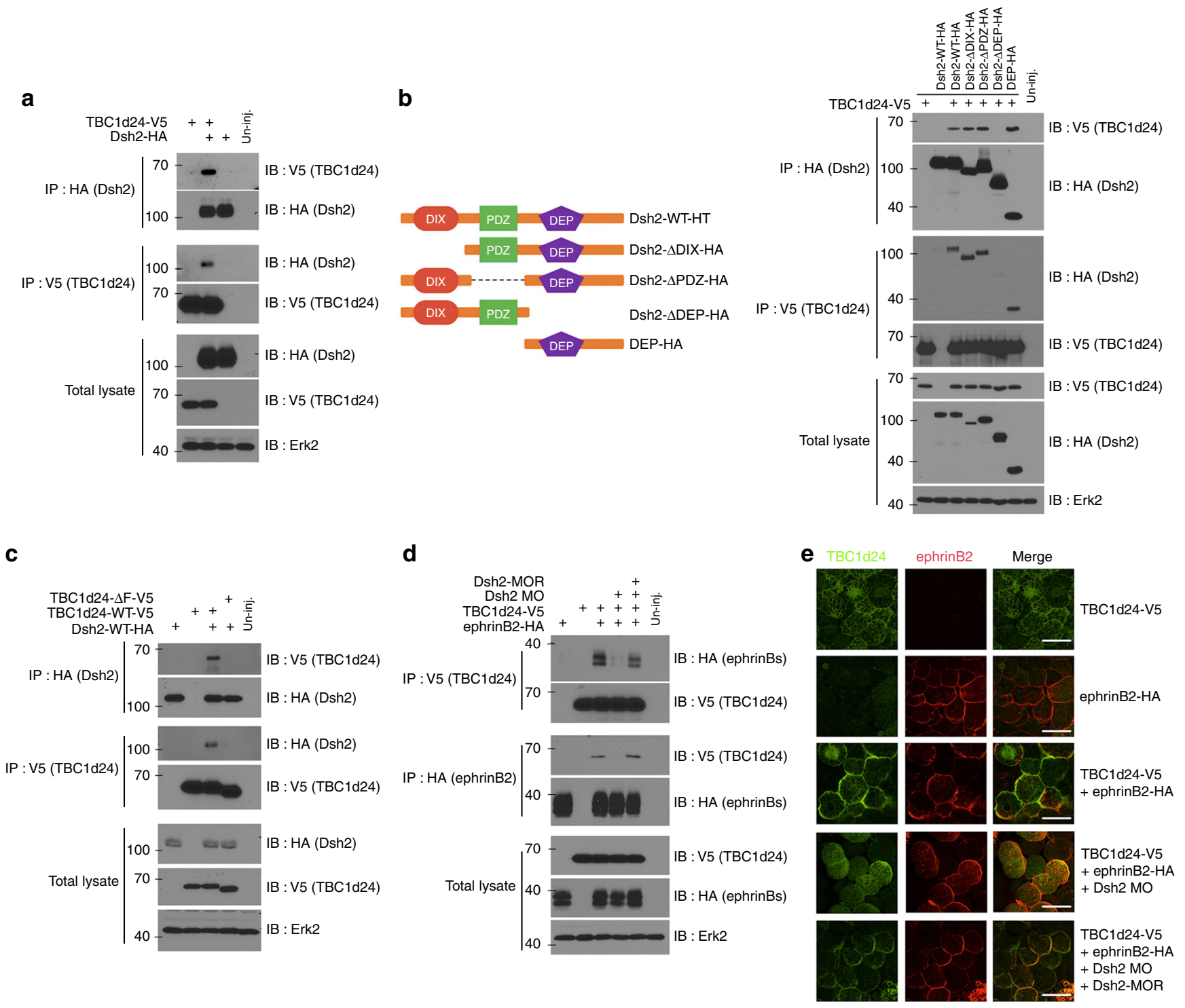

Fig. 2 Dsh mediates the ephrinB2-TBC1d24 interaction. a Co-IP using gastrula embryos injected with RNA encoding TBC1d24-V5 and Dsh-HA (500 pg) shows that TBC1d24 binds to Dsh. $\mathbf{b}$ Illustration of the deletion mutants of Dsh. Co-IP using gastrula embryos injected with RNA encoding TBC1d24-V5 and domain deletion mutants of Dsh-HA shows that the DEP domain of Dsh associates with TBC1d24. c Co-IP using gastrula embryos injected with RNA encoding Dsh-HA and wild-type TBC1d24-V5 or the mutant TBC1d24- $\Delta \mathrm{F}$ shows that the $\mathrm{F}$ region is required for an interaction with Dsh. $\mathbf{d}$ IP using gastrula embryos injected with RNA for TBC1d24-V5 and Dsh-HA along with Dsh2 morpholino oligonucleotides (Dsh2-MO) shows that knockdown of Dsh2 suppresses the interaction between ephrinB2 and TBC1d24, and this is rescued by the expression of MO-resistant Dsh2 (Dsh2-MOR). e IF of ectoderm from the embryos injected with the indicated MOs and RNAs shows that ephrinB2 (red) induces membrane localisation of TBC1d24 (green). This localisation is dramatically reduced by knockdown of Dsh2 and also rescued by introduction of Dsh2-MOR RNA. Bar, $50 \mu \mathrm{m}$

can regulate signalling partner associations and reverse signalling through the intracellular domain of ephrinBs ${ }^{36}$. Of note, phosphorylation of ephrinB1 is known to cause the disengagement of its physical association with $\mathrm{Dsh}^{40,42}$. Thus we tested whether the ephrinB2/TBC1d24 association is also regulated by tyrosine phosphorylation, as would be expected if Dsh were the mediator of the interaction. When a constitutively active FGFR1 (caFGFR) or an EphB4 receptor lacking the tyrosine kinase domain (EphB4$\Delta \mathrm{C}$ ) was expressed in Xenopus embryos, ephrinB2 tyrosine phosphorylation was induced (Fig. 3a, b). Interestingly, immunoprecipitation and western blot analysis of ephrinB2 indicates that phosphorylation causes a marked reduction in its interaction with TBC1d24 (Fig. 3a, b), whereas a mutant ephrinB2 with all five conserved tyrosines changed to phenylalanine showed no alteration in its interaction with $\mathrm{TBC} 1 \mathrm{~d} 24$ (Fig. 3a). Together, these data indicate that phosphorylation induced by either binding of EphB or activation of the FGFR can disrupt the association between ephrinB2 and TBC1d24.

To test whether contact between ephrinB2 and its cognate receptor also results in inhibiting ephrinB2-induced membrane localisation of $\mathrm{TBC} 1 \mathrm{~d} 24$, ephrinB2 and green fluorescent protein (GFP)-TBC1d24 RNA were injected into one blastomere of a twocell-stage embryo. The other blastomere was injected with membrane-red fluorescent protein (membrane-RFP) RNA singly or along with EphB4- $\Delta \mathrm{C}$ RNA. The animal ectoderm region was dissected and observed under confocal microscopy at stage 10 (gastrula). TBC1d24 protein localised to the membrane at the point of cell-cell contact between the cells expressing both ephrinB2 and GFP-TBC1d24 and the cells expressing the membrane-RFP alone, as expected (Fig. 3c). In contrast, membrane localisation of TBC1d 24 
a

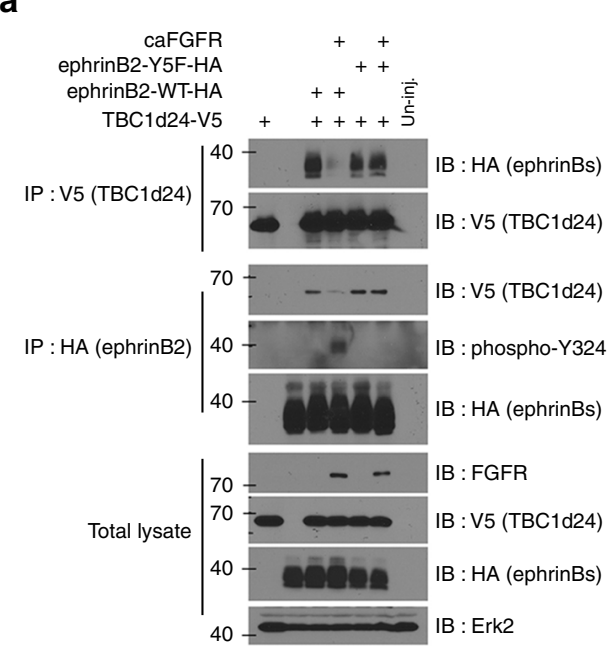

C

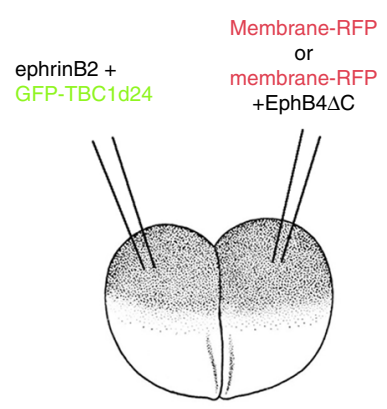

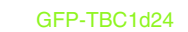
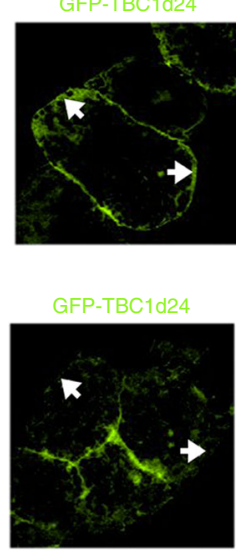

Membrane-RFP

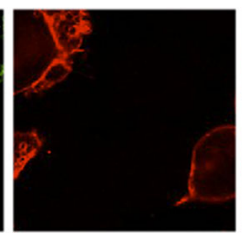

Membrane-RFP $+\mathrm{EphB} 4 \Delta \mathrm{C}$

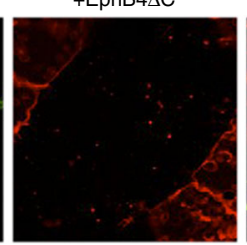

b EphB4- $\Delta$ C-Flag

$+$

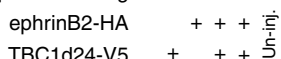

IP : V5 (TBC1d24) $\mid \begin{array}{ll}40- & \text { IB : HA (ephrinBs) } \\ 70- & \text { IB : V5 (TBC1d24) }\end{array}$

IP : HA (ephrinB2) $\mid \begin{array}{ll}70- & \text { IB : V5 (TBC1d24) } \\ 40- & \text { IB : HA (ephrinBs) }\end{array}$
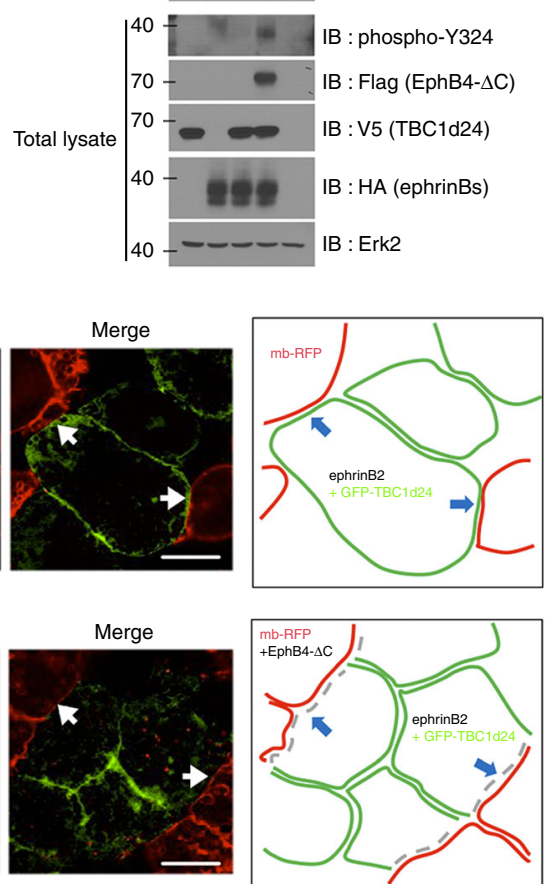

Fig. 3 The interaction between ephrinB2 and TBC1d24 is disrupted by tyrosine phosphorylation. a Co-IP using gastrula embryos injected with RNA for TBC1d24-V5 and wild-type ephrinB2-HA or the ephrinB2 tyrosine mutant (Y5F) along with a constitutively active mutant of FGFR1 (caFGFR; 250 pg) shows that ephrinB2 phosphorylation suppresses the interaction between TBC1d24 and ephrinB2. b Co-IP using gastrula embryos injected with RNA encoding TBC1d24 and ephrinB2 wild type along with EphB4- $\Delta C$ (having a truncated cytoplasmic domain) (1 ng) shows that the interaction between ephrinB2 and the ectodomain of its cognate receptor EphB4 inhibits ephrinB2-TBC1d24 binding. c IF of ectoderm tissue excised from the embryos injected with the indicated RNAs shows that membrane localisation of GFP-TBC1d24 (green) in ephrinB2-expressing cells is dramatically reduced at the site of cell-cell contact with cells expressing both EphB4- $\Delta$ C and membrane-RFP (red). However, membrane localisation of GFP-TBC1d24 is unaffected by contact with only membraneRFP-expressing cells. The white arrows indicate cell-cell contact regions. The cartoon depicts areas in the merged image. Bar, $20 \mu \mathrm{m}$

along the cell-cell contact region was significantly decreased when cells expressing both ephrinB2 and GFP-TBC1d24 were in contact with cells expressing EphB4- $\Delta$ C along with membrane-RFP (Fig. 3c). Although tyrosine phosphorylation disrupts the interaction between ephrinB2 and the Dsh/TBC1d24 complex, it was still possible that, upon contact with EphB4- $\Delta$ C-expressing cells, ephrinB2 is endocytosed and leads to loss of $\mathrm{TBC} 1 \mathrm{~d} 24$ membrane localisation. However, expression of an ephrinB2 tyrosine to phenylalanine substitution mutant (ephrinB2-Y2F) that precludes phosphorylation of the two tyrosines necessary for disengagement of $\mathrm{Dsh}^{40}$ maintained the membrane localisation of GFP-TBC1d24 at the site of cell-cell contact with EphB4- $\Delta \mathrm{C}$ (Supplementary Fig. 1e). Taken together, our results demonstrate that $\mathrm{TBC} 1 \mathrm{~d} 24$ interacts with ephrinB2 via Dsh and is localised to the membrane. However, upon tyrosine phosphorylation, the association between ephrinB2 and TBC1d 24 is disrupted leading to re-localisation of TBC1d 24 from the plasma membrane to the cytoplasm.

Loss of TBC1d24 function causes CNC cell migration defects. EphrinB2 is expressed in CNC cells of vertebrate embryos, including mouse, chick and frog $22,24,27,48$. We performed wholemount in situ hybridisation (WISH) with probes for TBC1d24 and found that its expression overlaps with ephrinB2 in $\mathrm{CNC}$ cells and somites (Fig. 4a). To define the functional role of TBC1d24 in embryonic CNC, we designed a specific MO against TBC1d24 (TBC1d24 MO) and confirmed the ability of the MO to block translation of exogenous protein (Supplementary Fig. 2a). Interestingly, embryos injected at the two-cell stage with TBC1d24 MO show reduced pharyngeal pouch formation that is a typical phenotype induced by CNC cell migration defects at the tailbud stage (Supplementary Fig. 2b) ${ }^{49}$. To exclude possible indirect causes for the phenotype such as improper gastrulation or delayed neural tube closure, embryos were injected in the D.1.2 blastomere of 16-cell-stage embryos, which restricts the MO to the region giving rise to neural crest (Supplementary Fig. 2c). In these experiments, one side of the embryo has the progeny cells harbouring the MO, while the other side does not, acting as a control. WISH was performed using a probe for Twist, used as a marker for migrating neural crest. In the early neurula-stage embryos, neural crest induction was not altered by TBC1d $24 \mathrm{MO}$ 
a

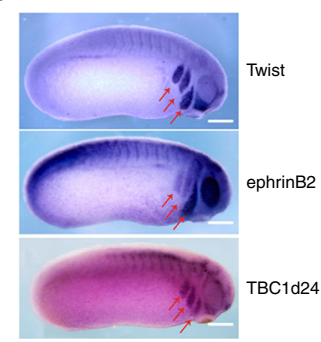

b

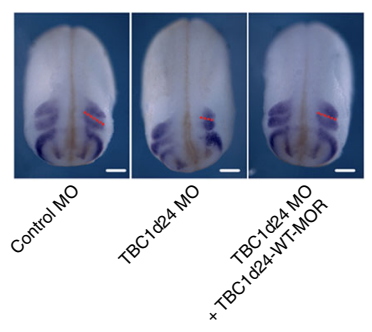

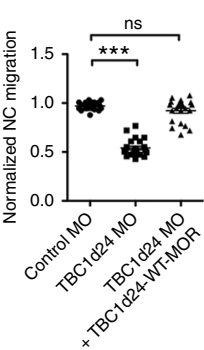

C

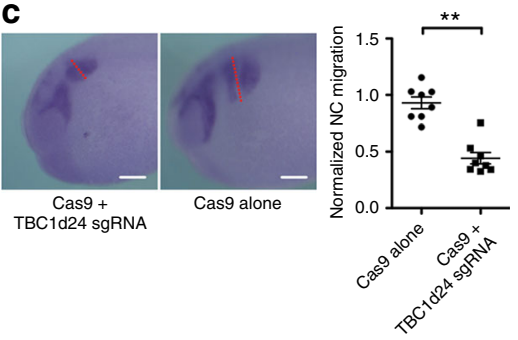

d
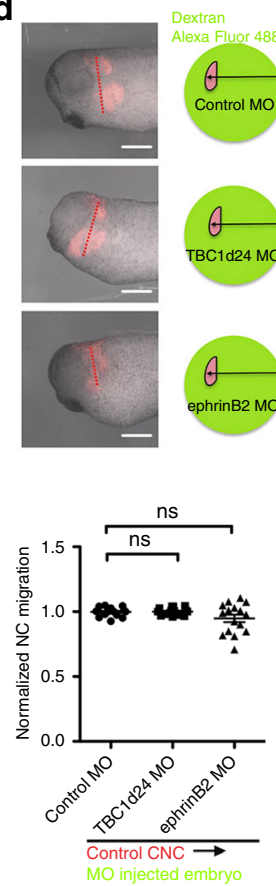
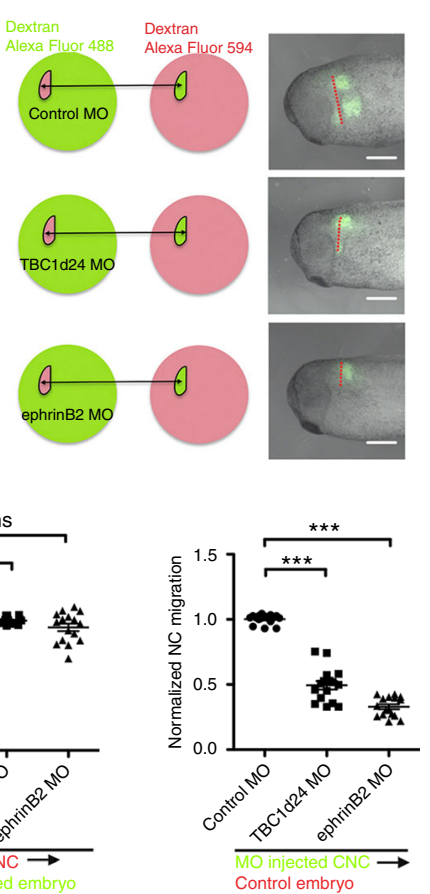

e

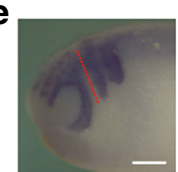

Control MO

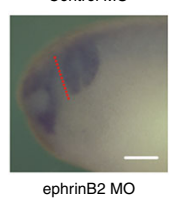

ephrinB2 MO + ephrinB2-WT-MO

f

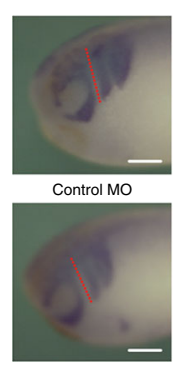

$\mathrm{TBC} 1 \mathrm{~d} 24 \mathrm{MO}$ $\begin{array}{r}\text { TBC1d24 MO } \\ + \text { TBC1d24-WT-MO } \\ \hline\end{array}$

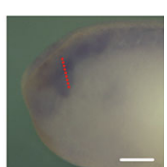

ephrinB2 MO
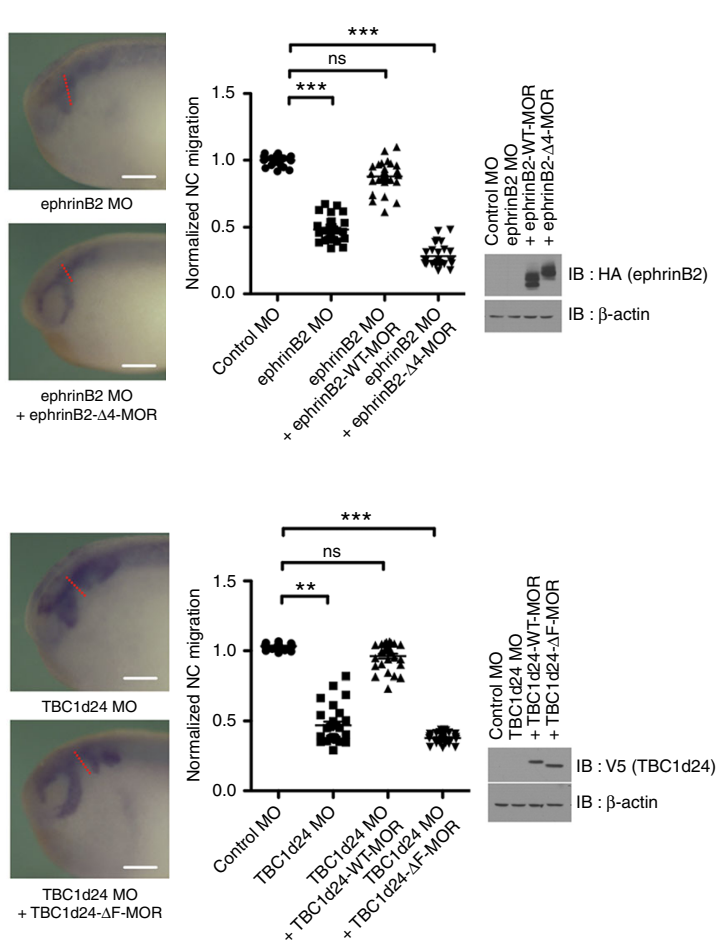

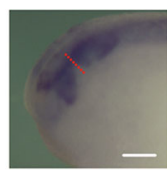

TBC1d24 MO

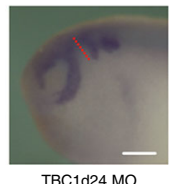

TBC1d24 MO
+ TBC1d24- $-\mathrm{F}-\mathrm{MOR}$

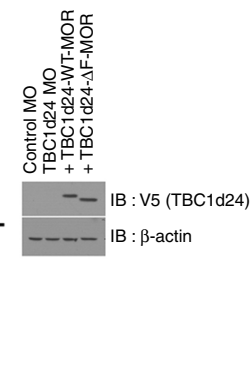

Fig. 4 EphrinB2-TBC1d24 interaction regulates CNC cell migration. a Spatial expression pattern of Twist, ephrinB2 and TBC1d24. WISH of stage 24 embryos with probes for twist (a CNC marker), ephrinB2 and TBC1d24 (red arrows indicate CNC). Lateral view, anterior is right. b WISH using the Twist antisense probe in embryos injected with Control MO or TBC1d24MO alone or along with TBC1d24 MO-resistant RNA. MOs and/or RNAs were injected into the D.1.2 blastomere. The dotted red line denotes distance of CNC migration on the injected side of the tailbud stage embryo. Anterior/dorsal view. Quantification with non-parametric ANOVA (Kruskal-Wallis test), $P<0.0001$. c WISH using the Twist antisense probe in FO embryos injected with sgRNA for TBC1d24 and/or Cas9 protein. Dotted red line denotes distance of CNC migration. Quantification with Mann-Whitney test: ${ }^{\star \star} P=0.0013$, two-tailed). $\mathbf{d}$ Green fluorescein dextran (GFD)-labelled neural crest explant tissue from control, TBC1d24 or ephrinB2-depleted embryos was exchanged with normal neural crest explant tissue from red fluorescein dextran (RFD)-labelled embryos at stage 20 as indicated in the schematic (left). At the tailbud stage (right), it is observed that depletion of ephrinB2 or TBC1d24 causes neural crest cell migration defects in a tissue-autonomous manner. Quantification with one-way ANOVA, $P<0.0001$. e WISH using the Twist antisense probe in the embryos injected with ephrinB2 MO alone or with MO-resistant wild-type or $\triangle 4$ ephrinB2 mutant RNA as indicated. Dotted red line denotes distance of CNC migration. Quantification with non-parametric ANOVA (Kruskal-Wallis test), $P<0.0001$. Western blot shows the similar indicated protein expression. $\mathbf{f}$ WISH using twist probe in embryos injected with the indicated MOs and RNAs. The red arrow indicates the CNC migration differences between embryos. Western analysis shows that mutant and wild-type MO-resistant RNAs yield similar levels of protein expression. Quantification with non-parametric ANOVA (Kruskal-Wallis test), P<0.0001; Bar, 200 $\mu \mathrm{m}$. All histograms and scatterplots represent mean \pm s.e.m from three biological repeats; Dunnett's multiple comparison; ${ }^{\star \star} P<0.01$ and ${ }^{\star \star \star} P<0.001$, ns: no statistical differences between groups

and the muscle marker, MyoD, was also unchanged (Supplementary Fig. 2d). Of particular importance, in the tailbud-stage embryos, neural crest cell migration was suppressed in a MO dose-dependent manner (Fig. $4 \mathrm{~b}$ and Supplementary Fig. 2e). This was also confirmed using a probe for Slug, another neural crest-specific marker (Supplementary Fig. 2f). The CNC migration defects were rescued by injection of MO-resistant wild-type TBC1d24 RNA, verifying that the defect was specific to reduced TBC1d24 (Fig. 4b and Supplementary Fig. 4f). To further validate the loss-of-function phenotype conferred by the TBC1d24 MO, we employed a knockout strategy using CRISPR/Cas9
(Supplementary Fig. 2g). TBC1d24 CRISPR-targeted embryos also showed similar CNC migration defects (Fig. 4c).

The ephrinB2 ligand is a transmembrane protein that is capable of bi-directional signalling, which prompted a test of whether TBC1d24- or ephrinB2-mediated effects were tissue autonomous or non-autonomous. At the 16-cell stage, TBC1d24 $\mathrm{MO}$ or ephrinB2 $\mathrm{MO}$ were injected along with a fluorescent tracer into the D.1.2 blastomere and incubated until stage 20 (when neural crest is migratory). Neural crest tissue was excised and transferred to a recipient embryo where the neural crest tissue had been previously removed (Fig. 4d and Supplementary 
Fig. 2h). Neural crest cells lacking either TBC1d24 or ephrinB2 failed to migrate in the wild-type-recipient embryos, whereas wild-type neural crest cells migrate normally even in recipient embryos in which TBC1d 24 or ephrinB2 were depleted in the surrounding tissue (Fig. 4d). Moreover, morphant CNC explants injected with MO-resistant wild-type TBC1d24 or ephrinB2 RNA, respectively, had ventral migration of CNC restored (Supplementary Fig. 2h). These results strongly imply that ephrinB2 and TBC1d24 regulate neural crest cell migration in a tissue autonomous manner.

An ephrinB2/TBC1d24 interaction is required for CNC migration. Since our biochemical and cell biological data established that ephrinB2 interacts with $\mathrm{TBC} 1 \mathrm{~d} 24$ and controls the localisation of TBC1d24, we tested whether the TBC1d24 and ephrinB2 interaction plays a significant role in CNC migration. Both ephrinB2 and TBC1d24 morphant embryos displayed abnormal CNC cell migration, which was rescued by expressing their wild-type counterparts (Fig. 4e, f, respectively). However, the expression of ephrinB2- $\Delta 4$, which cannot associate with $\mathrm{TBC} 1 \mathrm{~d} 24$, or the expression of $\mathrm{TBC} 1 \mathrm{~d} 24-\Delta \mathrm{F}$, which cannot interact with ephrinB2, failed to rescue their affiliated morphant defects (Fig. 4e, f, respectively). Western blot analysis shows that the expression of the interaction mutants was similar to their wild-type counterparts (Fig. 4e, f). These observations suggest that ephrinB2 and TBC1d24 interaction plays a critical role in proper neural crest cell migration.

Rab35 interacts with TBC1d24 and regulates CNC migration. Skywalker is the Drosophila homologue of TBC1d24 that functions as a RabGAP for Rab35 $5^{50}$. TBC1d24 is also known to regulate neuronal migration and maturation by negatively regulating activation of Arf6, which is involved in membrane trafficking ${ }^{51}$. In addition, a number of other Rabs are potential candidates for affecting or regulating CNC migration: Rab11 is associated with E-cadherin recycling in mammals and $\mathrm{Fly}^{52}$, while Rab5 and Rab7 have been implicated in lysosomal targeting of Ecadherin ${ }^{53}$. A limited Co-IP screen was performed with Arf6 and several Rabs in Xenopus embryos, and Xenopus TBC1d24 interacted with Rab5 and Rab35, but Arf6 showed only a weak interaction (Supplementary Fig. 3a). Co-IP analysis of lysates from the embryos expressing TBC1d24 mutants harbouring small deletions within the conserved TBC domain (delineated in Fig. 1c) $)^{50,51}$ indicated that the TBC1d24- $\Delta \mathrm{D}$ mutant showed a significant reduction in the ability to bind Rab35, while the TBC1d24- $\Delta \mathrm{F}$ mutant (which is unable to interact with ephrinB2) interaction was unchanged (Supplementary Fig. 3b). Unlike wildtype TBC1d24, expression of the TBC1d24- $\Delta \mathrm{D}$ mutant failed to rescue CNC migration in TBC1d24 morphant embryos (Supplementary Fig. 3c). These findings strongly suggest that the binding of Rab35 to TBC1d24 plays a key role in proper CNC migration.

TBC1d24 modulates CIL via regulating E-cadherin. One major contributor to the appropriate migration of $\mathrm{CNCs}$ is a process known as CIL, in which cells halt migration in response to contact with another cell. In CNC cells, homotypic collisions or contact between $\mathrm{CNC}$ cells leads to retraction of cell protrusions, repolarisation and cells move away from each other. To examine how loss of TBC1d24 function causes neural crest cell migration defects, we dissected migratory neural crest tissue from stage 20 embryos that were previously injected with MOs and RNAs, as indicated in Fig. 5a. The neural crest tissue explants injected with either green or red fluorescent dextran (GFD or RFD, respectively; as a lineage tracer) were placed on fibronectin-coated plates and subjected to an invasion assay that tests whether the explants have lost CIL and thus leads to one explant mixing or invading into the other explant. Unlike control MO-injected neural crest tissue (green), TBC1d24-depleted neural crest tissue (green) invaded the uninjected neural crest tissue (red), demonstrating that homotypic CIL is compromised (Fig. 5a). Coinjecting wild-type TBC1d24 RNA into the TBC1d24-depleted explant blocked invasion and restored CIL (Fig. 5a). EphrinB2depleted neural crest tissue (green) also invaded the uninjected neural crest tissue (red), which was rescued by expressing the wild-type form (Supplementary Fig. 3d). However, the expression of ephrinB2- $\Delta 4$, which cannot bind to TBC1d24, failed to block the invasive behaviour (Supplementary Fig. 3d). Neither TBC1d24- $\Delta \mathrm{D}$ nor TBC1d24- $\Delta \mathrm{F}$ expression in TBC1d24-depleted neural crest explants (green) rescued CIL, as evidenced by the invasive behaviour (Fig. 5a). These data strongly indicate that $\mathrm{TBC} 1 \mathrm{~d} 24$ requires an interaction with both Rab35 and ephrinB2 to preserve homotypic CIL.

An additional test of whether these interactions are critical to promote homotypic CIL was initiated. An ex vivo CNC cell migration assay was performed on similarly injected embryos whose neural crest was excised and dissociated to examine singlecell migration. In this assay, homotypic collisions between CNC cells that display CIL should lead to retraction of cell protrusions, repolarisation and cell migration away from each other. CNC cells from the control MO-injected embryos and TBC1d24depleted cells that had not collided with other CNC cells showed no significant differences in migration direction or speed (Fig. 5b). This was also true of those $\mathrm{CNC}$ cells expressing wild-type $\mathrm{TBC} 1 \mathrm{~d} 24, \mathrm{TBC} 1 \mathrm{~d} 24-\Delta \mathrm{D}$ or $\mathrm{TBC} 1 \mathrm{~d} 24-\Delta \mathrm{F}$ interaction mutants (Fig. 5b). In contrast, there was a significant increase in contact time during cell collisions between CNC cells lacking TBC1d24 (Fig. 5c; Supplementary movie 1), which is a hallmark of loss of $\mathrm{CIL}^{54}$. Consistent with the invasion assays, re-expression of wildtype TBC1d24 in TBC1d24-depleted cells led to a restoration of normal contact times between CNC cells (Fig. 5c; Supplementary movie 1). Of note, in the TBC1d24-depleted cells, expression of the TBC1d24 mutants that are unable to interact with Rab35 or ephrinB2 failed to restore normal contact times (Fig. 5c; Supplementary movie 1). Taken together, these results suggest that $\mathrm{TBC} 1 \mathrm{~d} 24$ regulates homotypic CIL during neural crest cell migration.

It has been reported that $\mathrm{CNC}$ cells expressing E-cadherin remain in contact, while cells expressing $\mathrm{N}$-cadherin undergo cell repolarisation and repulsion ${ }^{3}$. It was shown that overexpression of E-cadherin inhibits CIL behaviour after cell-cell collision ${ }^{3}$, and this is very similar to our results with the TBC1d24-depleted CNC cells (Fig. 5c; Supplementary movie 1). Furthermore, Rab35, which is a substrate of TBC1d24, is known to regulate E-cadherin recycling in the mammalian cell lines ${ }^{31,55}$. To assess effects on Ecadherin levels, neural crest tissue from the embryos injected with RNA encoding mutants of the three potential Arf/Rabs (Arf6, Rab5 and Rab35) that interact with TBC1d24 (Supplementary Fig. 3a) were dissected at stage 20 and immunostained for Ecadherin (Supplementary Fig. 3e). GFP control-expressing CNC cells displayed a low level of E-cadherin, as did those expressing a constitutively active or inactive Arf6. Overexpression of a constitutively active form of Rab35 (Rab35 Q67N) increased Ecadherin levels, while the inactive form of Rab35 (Rab35 S22N) reduced E-cadherin levels in Xenopus CNC cells (Supplementary Fig. 3e). Not surprisingly, the opposite result was observed with Rab5 mutants (Supplementary Fig. 3e). Rab5 is generally involved in early endocytosis from the membrane ${ }^{56}$, while Rab35 is mainly concerned with recycling to the membrane. Thus E-cadherin levels were only elevated in the active Rab35-expressing or inactive Rab5-expressing CNC cells. 
a

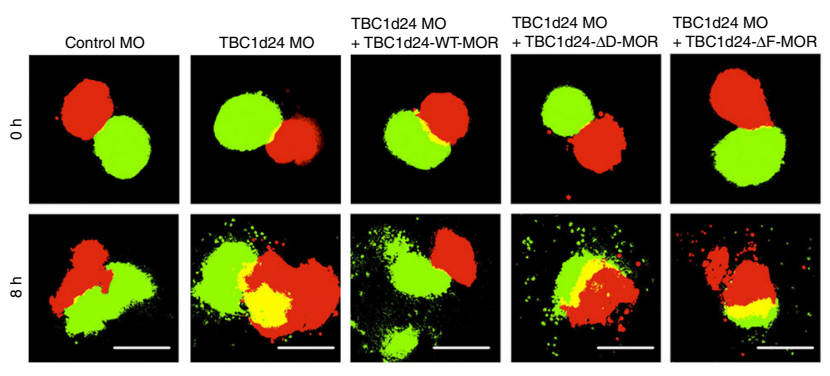

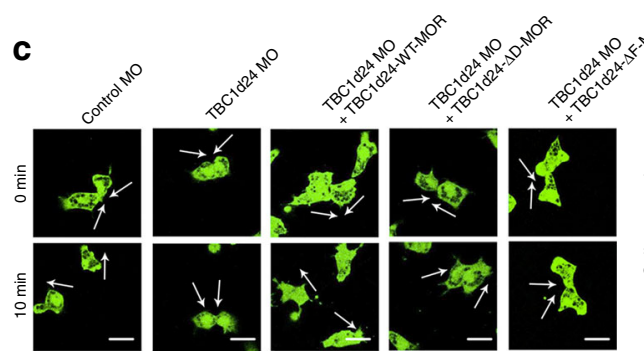

d

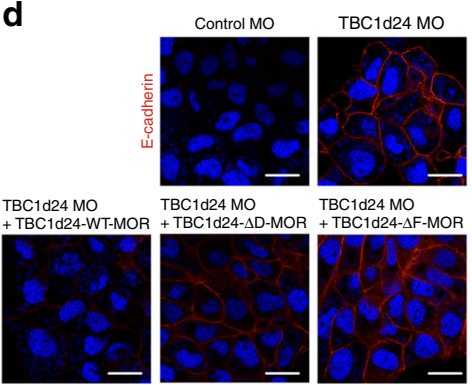

e

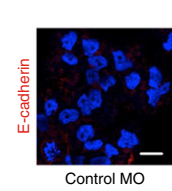

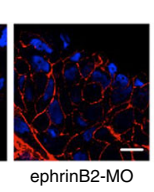

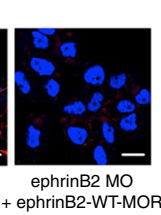

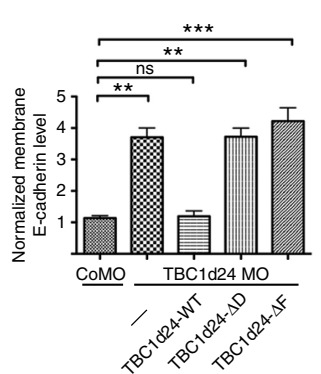

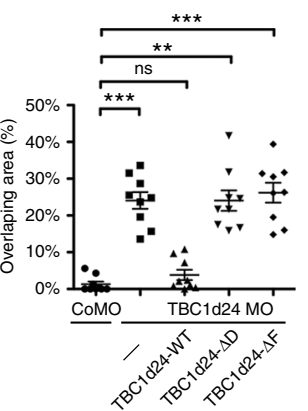

b

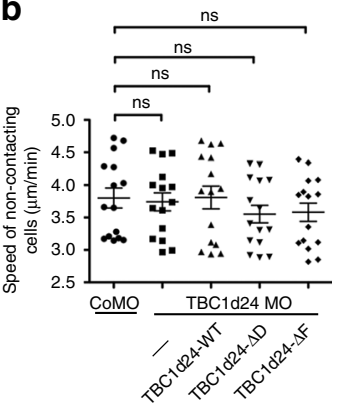

$\mathbf{f}$
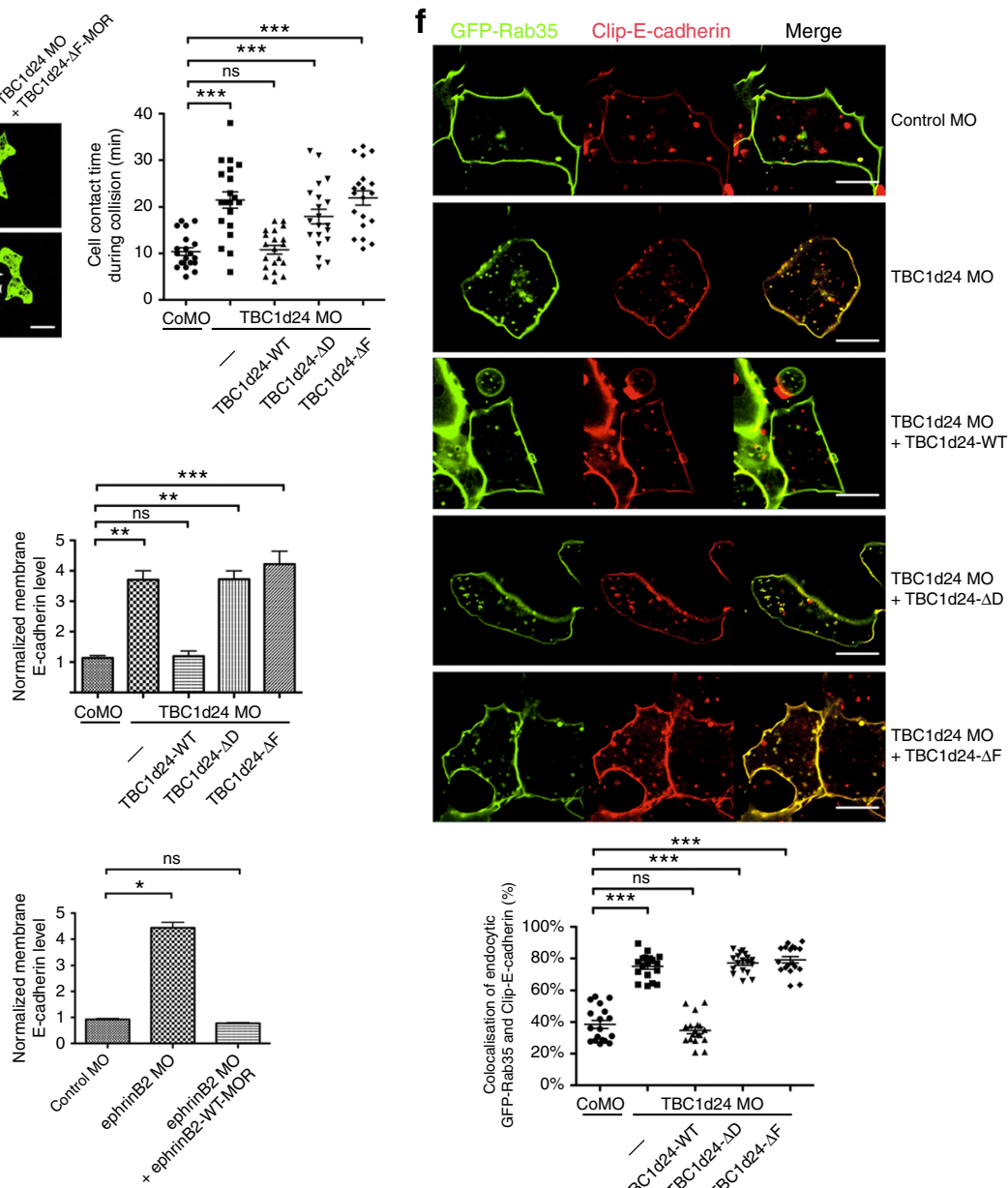

Fig. 5 TBC1d24 modulates CIL via regulating E-cadherin. a Homotypic invasion assay. CNC explants injected with control MO or TBC1d24 MO alone or with the indicated TBC1d24 MO-resistant WT or mutant RNA and juxtaposed with RFD-injected CNC explants. Invasion is determined by the amount of explant overlap (yellow). Quantification with non-parametric ANOVA (Kruskal-Wallis test), $P<0.0001 ; B a r, 200 \mu$ m. b Histogram depicts ex vivo analysis of the speed of non-contacting CNC cells $(\mu \mathrm{m} / \mathrm{min}$ ) injected with the indicated MOs and RNAs. Quantification with one-way ANOVA, $P=0.8846$. $\mathbf{c}$ Ex vivo migrating neural crest cell contact assay. The white arrows in the IF still images (from Supplementary Movie 1) of cells from CNC explants injected with the specified MOs and RNAs indicate the direction of migration at the indicated time of collision. The histogram indicates contact time during collisions of cells injected with control or TBC1d24 MOs alone or with the indicated TBC1d24 RNAs. Data represents means \pm s.e.m from four biological repeats. Quantification with one-way ANOVA, P<0.0001; Bar, $20 \mu \mathrm{m}$. d IF of CNC tissue using anti-E-cadherin Ab (red) from the embryos injected with control or TBC1d24 MOs alone or with the indicated TBC1d24 RNAs. Histogram represents relative E-cadherin levels. Quantification with non-parametric ANOVA (Kruskal-Wallis test), P $<0.0001 ;$ Bar, $20 \mu \mathrm{m}$. e IF of CNC tissue with an E-cadherin Ab (red) from the embryos injected with control or ephrinB2 MOs alone or with MO-resistant ephrinB2 RNA. Histogram represents relative E-cadherin levels. Quantification with non-parametric ANOVA (Kruskal-Wallis test), $P<0.0001$; Bar, $20 \mu \mathrm{m}$. f IF of CNC explants expressing GFP-Rab35 (green) and Clip-tagged E-cadherin (red) also injected with control or TBC1d24 MOs alone or with the indicated TBC1d24 RNAs. Co-localisation displayed as yellow area. Histogram represents the percentage of overlapping signal in endocytic vesicles. Quantification with one-way ANOVA, $P<0.0001$. Bar, $10 \mu \mathrm{m}$. All histograms and scatterplots represent mean $\pm \mathrm{s}$. e.m from three biological repeats unless otherwise indicated; Dunn's multiple Comparison, ${ }^{\star} P<0.05,{ }^{\star \star} P<0.01$ and ${ }^{\star \star \star} P<0.001$, ns: no statistical differences between the groups 
Since elevated levels of E-cadherin are associated with inhibition of CIL in CNC cells ${ }^{3}$, we tested whether depletion of ephrinB2 or TBC1d24 also yields an increase in E-cadherin levels. Both TBC1d24 MO and ephrinB2 MO bearing neural crest tissue displayed elevated E-cadherin levels at the cell membrane, and this increase was blocked by re-expression of the wild-type counterparts of TBC1d24 or ephrinB2 (Fig. 5d, e). In the TBC1d24-depleted CNC cells, expression of the inactive Rab35 blocked the enhanced E-cadherin expression, while inactive Rab5 did not, supporting the data indicating that TBC1d24 exerts its regulation of E-cadherin through Rab35 (Supplementary Fig. 3f). N-cadherin levels did not show any significant changes in TBC1d24- or ephrinB2-depleted neural crest tissue (Supplementary Fig. 3g). Expression of the Rab35 or ephrinB2 interaction mutants of TBC1d24 failed to decrease the enhanced E-cadherin levels observed with the loss of TBC1d24 (Fig. 5d), strongly suggesting that the interaction between TBC1d24 and these proteins is critical for regulating surface E-cadherin levels.

A more direct assessment of whether $\mathrm{TBC} 1 \mathrm{~d} 24$ is responsible for maintaining a low level of recycling of E-cadherin to the CNC membranes was performed. We expressed Clip-tagged Ecadherin that was later incubated in a cell non-permeable substrate to label surface E-cadherin. Neural crest tissue bearing the TBC1d24 MO showed a noticeably higher abundance of vesicles and plasma membrane regions that were positive for both Rab35 and E-cadherin (observed as yellow in the merged image in Fig. 5f; Supplementary movie 2), when compared to the control MO-injected CNC (observed as more distinct red and green areas in the merged image in Fig. 5f; Supplementary movie 2). This data is indicative of greater co-localisation of Rab35 and Ecadherin in the absence of $\mathrm{TBC} 1 \mathrm{~d} 24$, as would be expected if more E-cadherin is cycling to the membrane. When wild-type TBC1d24 was re-expressed in the TBC1d24 MO bearing CNC cells, co-localisation between Rab35 and E-cadherin was reduced, similar to control MO-injected CNC (Fig. 5f; Supplementary movie 2). Unlike wild-type $\mathrm{TBC} 1 \mathrm{~d} 24$, re-expressing either of the interaction mutants (TBC1d24- $\Delta \mathrm{D}$ or $\mathrm{TBC} 1 \mathrm{~d} 24-\Delta \mathrm{F}$ ) failed to restore the separate localisation of Rab35 and E-cadherin (Fig. 5f; Supplementary movie 2). In addition, ephrinB2 knockdown significantly increased the levels of co-localisation between Rab35 and E-cadherin when compared the control MO-injected CNC (Supplementary Fig. 3h; Supplementary movie 3), which was inhibited upon re-expression of wild-type ephrinB2. However, expression of ephrinB2- $\Delta 4$, which cannot associate with TBC1d24, failed to decrease the co-localisation (Supplementary Fig. 3h; Supplementary movie 3). Taken together, our results indicate that the interaction of $\mathrm{TBC} 1 \mathrm{~d} 24$ with ephrinB2 and Rab35 is critical for regulating E-cadherin recycling.

EphrinB2 and EphB4 regulate proper CNC cell migration. Having found that overexpression of EphB4 disrupts the ephrinB2/TBC1d24 interaction (Fig. 3b, c), we examined how the $\mathrm{Eph} / \mathrm{ephrin}$ interaction may regulate proper CNC cell migration. A WISH survey of potential cognate Eph receptors showed that EphB4 and EphB1 transcripts were found in the tissue that borders the neural crest stream, whereas EphB2 is expressed within the neural crest cells, much like ephrinB2 (Fig. 6a). Previous studies demonstrate that ephrinB2 displays more robust interactions with EphB4 than EphB2 ${ }^{57}$, and our Co-IP results confirm this trend (Fig. 6b). To assess whether binding to the extracellular domain of specific cognate EphB receptors affects the ephrinB2/TBC1d24 interaction, we introduced truncated versions of EphB1 or EphB2 or EphB4 (EphB1- $\Delta$ C, EphB2- $\Delta$ C, EphB4- $\Delta C$ ) into the embryos. These constructs lack the cytoplasmic domain to ensure that phosphorylation of ephrinB2 is induced by receptor binding and not by their kinase activity. The EphB4 receptor displayed the most prominent interaction with ephrinB2, followed by EphB1, and EphB2 being the weakest (Fig. 6b). Consistent with these binding preferences, EphB4 markedly disrupted the ephrinB2/TBC1d24 interaction in Co-IPs (Fig. 6b), and EphB2 had little effect (Fig. 6b). The biochemical, ex vivo and in vivo data strongly support a model where ephrinB2 interacts with TBC1d24 via Dsh, and this leads to proper localisation of Rab35 to regulate surface E-cadherin levels within the CNC cells. Moreover, upon contact with the cognate EphB4 receptor, tyrosine phosphorylation of ephrinB2 leads to disengagement of the complex (Supplementary Fig. 4a).

An ex vivo test of this concept was performed by treating neural crest explants that were plated on fibronectin with either clustered EphB4-Fc or Control-Fc. EphB4-Fc treatment significantly suppressed dispersion of $\mathrm{CNC}$ cells from the neural crest explant during a $12 \mathrm{~h}$ period, when compared to Control-Fctreated tissue (Supplementary Fig. 4b). As expected from the model (Supplementary Fig. 4a), EphB4-Fc treatment of CNC explants increased E-cadherin levels in CNC cells (Supplementary Fig. 4c). These data indicate that contact of ephrinB2-expressing neural crest cells with the EphB4 ectodomain inhibits CNC migration via increasing E-cadherin levels.

Proper neural crest cell migration is critically dependent upon the control of E-cadherin levels within the $\mathrm{CNC}^{21,58,59}$. To assess whether the regulatory mechanism established in ex vivo assays may also occur in vivo, we ectopically expressed EphB4- $\Delta \mathrm{C}$ in neural crest cells and examined the pattern of CNC migration through WISH by probing for Twist (Fig. 6c). The mis-expression of the EphB4- $\Delta \mathrm{C}$ protein considerably inhibited neural crest cell migration, when compared to the GFP RNA-injected controls (Fig. 6c). One prediction from the model (Supplementary Fig. 4a) is that loss of EphB4 expression in the tissue surrounding the CNC streams may lead to mixing of the streams as a result of increased E-cadherin levels. Thus we injected EphB4 MO that effectively blocks exogenous EphB4 expression (Supplementary Fig. 4d) into the D.1.2 blastomere to deplete EphB4 in the CNC and surrounding tissues (Fig. 6d). Consistent with the prediction, the $\mathrm{CNC}$ migration streams mixed in the EphB4-depleted embryos and not in the control MO-injected embryos (Fig. 6d). As a further test of the model, we transplanted GFP-expressing CNC cells into host embryos that harboured either control $\mathrm{MO}$ or EphB4 MO and examined cross-sections through the CNC streams (Fig. 6e). CNC cells transplanted into EphB4 MOinjected host embryos displayed a complete lack of collective cell migration, similar to CNC cells that were depleted of E-cadherin and transplanted into control MO-injected hosts (Fig. 6e). Another expectation from the model is that membrane localisation of TBC1d 24 would be disrupted in the normal developmental context when CNC tissue contacts the surrounding placode tissue where EphB4 is endogenously expressed. Therefore, we transplanted GFP-TBC1d24-expressing CNC into either wild-type or EphB4 MO-injected embryos (Fig. 6f). At the site of contact between CNC cells and surrounding placodal cells, the membrane localisation of TBC1d24 was markedly reduced (Fig. 6f). However, in a host embryo depleted of EphB4, clear membrane localisation of TBC1d24 was found at the site of contact between the CNC and placode (Fig. 6f; white arrow heads). Collectively, these data indicate that in CNC, ephrinB2 controls E-cadherin levels through the interaction with Dsh and TBC1d24. Furthermore, this interaction can be regulated in a tyrosine phosphorylation-dependent manner upon binding the cognate Eph receptor.

An ex vivo invasion assay was performed as an additional test of the influence of EphB4 on the interaction between CNC and placode tissue. GFP-expressing wild-type CNC explants were 

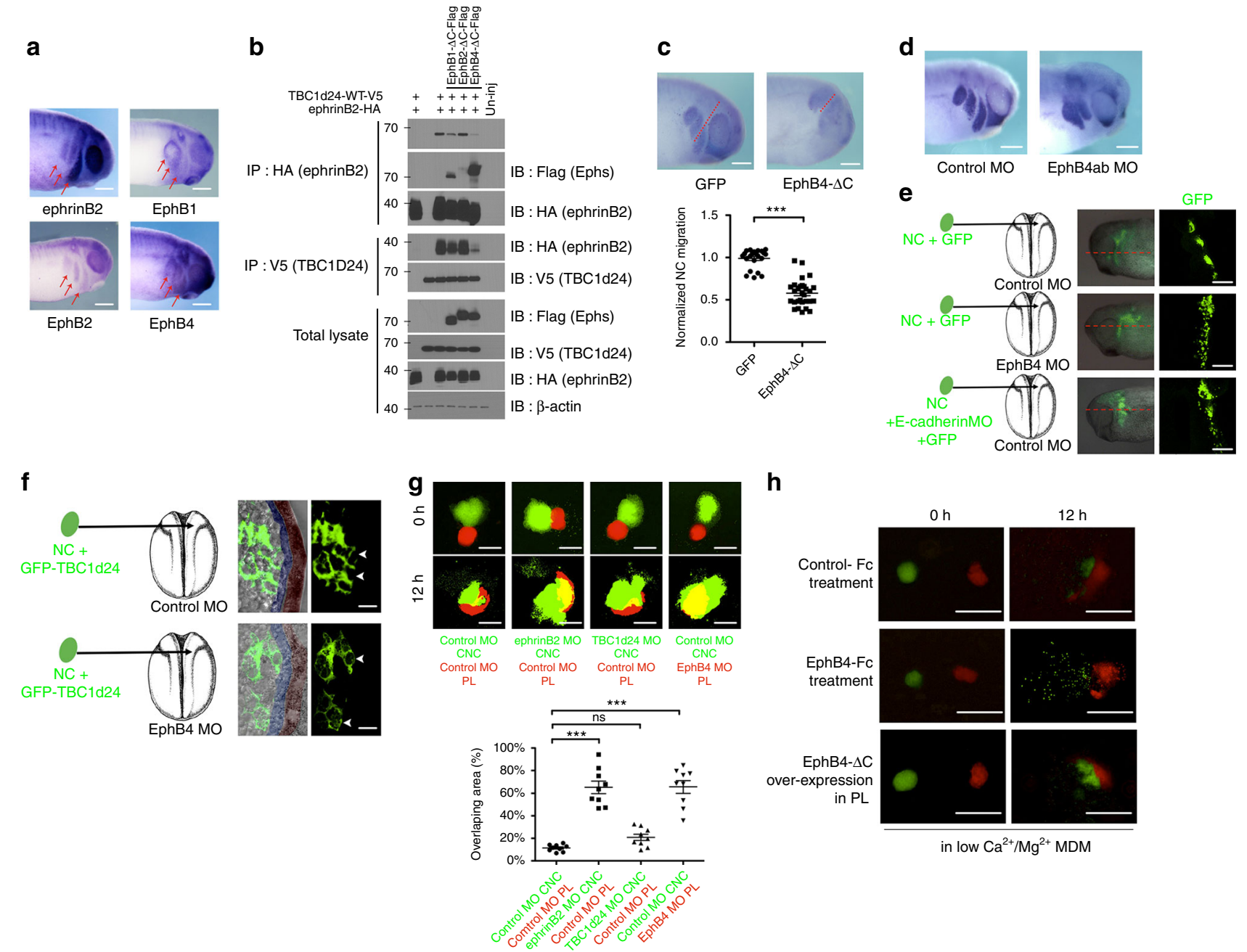

h

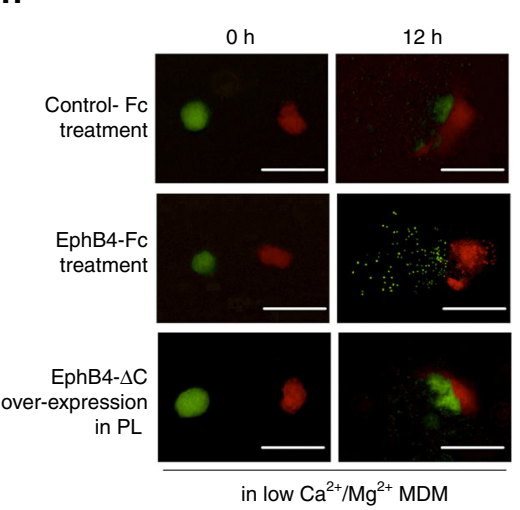

Fig. 6 EphrinB2 and its cognate Eph receptor, EphB4, regulate appropriate CNC cell migration. a WISH shows ephrinB2 and EphB2 expression in neural crest cells and EphB1 and EphB4 in boundary of CNC streams (red arrows). Bar, $200 \mu \mathrm{m}$. b Co-IP and western blot using gastrula embryos injected with TBC1d24 RNA and ephrinB2 wild-type RNA along with truncated receptor EphB1- $\Delta$ C, EphB2- $\Delta$ C or EphB4- $\Delta C$ RNAs (1 ng each). c, d WISH using Twist antisense probe in the embryos injected into the D.1.2 blastomere with the indicated MOs or RNAs (red dotted lines indicate extent of CNC migration). Histogram depicts relative CNC migration (quantification with Mann-Whitney test: ${ }^{\star \star \star} P=0.0004$, two-tailed). Bar, $200 \mu \mathrm{m}$. e Neural crest tissues injected with GFP RNA alone or along with E-cadherin MO were dissected and transferred into EphB4 MO or control MO-injected embryos as indicated. Confocal microscopic images of embryos sectioned along the red lines and the neural crest streams displayed. Bar, $200 \mu \mathrm{m}$. f GFP-TBC1d24 expressing neural crest tissues were dissected and transferred into EphB4 MO- or control MO-injected embryos. Embryos were sectioned and a magnified image shows that membrane localisation of GFP-TBC1d24 at the NC (neural crest)-PL (placode) contact site (the white arrow heads). Blue, placodes; brown, superficial ectoderm. Bar, $20 \mu \mathrm{m}$. $\mathbf{g}$ Heterotypic NC-PL invasion assay. Embryos were injected with the indicated MOs, and explants excised and placed juxtaposed. IF of GFD-labelled neural crest explants injected with the indicated MO (in green) juxtaposed to RFD-labelled placode explants injected with the indicated $\mathrm{MO}$ (red). Invasion of CNC into placode denoted by yellow area in image and depicted in histogram. Quantification with one-way ANOVA, $P$ < 0.0001; bar, $400 \mu \mathrm{m}$. h CNC chemoattraction assay. CNC (injected with GFD-green) or placode [(PL) injected with RFD-red] tissues were dissected at stage 20, placed $400 \mu \mathrm{m}$ apart and incubated in low $\mathrm{Ca}^{2+} / \mathrm{Mg}^{2+}$ medium for $12 \mathrm{~h}$ and treated with the indicated clustered Fc or injected with EphB4- $\Delta \mathrm{C}$ RNA and imaged. Bar, $400 \mu \mathrm{m}$. All histograms and scatterplots represent mean \pm s.e.m from three biological repeats; Dunn's multiple Comparison, ${ }^{\star \star \star} P<$ 0.001, ns: no statistical differences between the groups

placed adjacent to control wild-type RFP-expressing placode explants on a fibronectin-coated substrate. The CNC cells repulsed the placode tissue without mixing with or invading the tissue (Supplementary movie 3). However, when the placode tissue was depleted of EphB4, the CNC tissue contacted and subsequently invaded the EphB4-depleted placode tissue (Supplementary movie 4). These data clearly indicate that EphB4 in the placode is critical to maintain separation of the CNC and placode tissue, which is a consistent with the conventional EphB4/ephrinB2 repulsive activity ${ }^{60}$.
Although we have established that the ephrinB2/TBC1d24 interaction is critical for homotypic CIL within the CNC, it was less clear whether it has a role in heterotypic CIL (CNC/placode). Therefore, we performed neural crest tissue explant invasion assays on a fibronectin substrate using either GFD (neural crest) or RFD (placode) as lineage tracers. Unlike control CNC, ephrinB2-depleted CNC tissue invaded the placode explant (Fig. 6g). Of particular interest, TBC1d24-depleted neural crest tissue did not display significant invasive activity into the placode tissue (Fig. 6g), demonstrating that heterotypic CIL (CNC/ 
placode) is not compromised to a substantive degree. In contrast, EphB4 MO-containing placode tissue allows CNC explant invasion into the placode tissue (Fig. 6g). Together, these data indicate that $\mathrm{TBC} 1 \mathrm{~d} 24$ is not a major contributor to heterotypic CIL between CNC and placode, strongly suggesting that the repulsion between ephrinB2 in the $\mathrm{CNC}$ and $\mathrm{EphB} 4$ in the placode are critical for this process.

Eph-ephrinB reverse signalling is also known to regulate $G$ protein-coupled chemoattraction ${ }^{22}$. Thus we tested whether the EphB4/ephrinB2 interaction may regulate chemotaxis of CNC towards the placode. To this end, we excised neural crest explants that were injected with GFD and placode explants containing RFD. These CNC migratory-stage explants were placed near each other on fibronectin-coated plates in a low calcium media for 12 h. This low $\mathrm{Ca}^{2+} / \mathrm{Mg}^{2+}$ media allows an assessment of chemotaxis in the absence of effects on homotypic cell-cell adhesion that might be exerted by an increase in surface Ecadherin, resulting from EphB4-Fc treatment of these explants. Explants treated with control-Fc showed the expected directional movement of CNC explant cells towards the SDF-1-secreting placode explants ${ }^{11}$ (Fig. 6h, Supplementary Movie 5). Strikingly, EphB4-Fc treatment suppressed the directional migration of CNC cells towards the SDF-1-expressing placode and caused the CNC cells to move randomly (Fig. 6h, Supplementary movie 5). This is consistent with ephrinB2 within the CNC inhibiting the activity of the SDF-1 receptor (C-X-C chemokine motif receptor 4 (CXCR4)) upon engagement with the cognate $\mathrm{EphB}$ receptor, as has been shown for cerebellar granule cell movement ${ }^{61}$. Similar to control explants, CNC explants exhibited directional migration towards the EphB4- $\Delta \mathrm{C}$-expressing placode explants. This data indicate that the clustered soluble EphB4 treatment inhibits the ephrinB2-expressing CNC response to chemoattractant from the placode but does not exert an effect on the placode explant (Fig. 6h, Supplementary movie 5). Collectively, these data suggest that the ephrinB2/TBC1d24 interaction is critical to homotypic CIL but is not a major contributor to heterotypic CIL between the $\mathrm{CNC}$ and placode. Moreover, EphB4 expressed in the placode exerts its influence upon contact with ephrinB2-expressing CNC cells mainly through repulsive activity and the inhibition of the $\mathrm{CNC}$ response to chemoattractant.

\section{Discussion}

CIL is a crucial process for many essential embryological events, including collective cell migration and boundary formation, while loss of CIL plays a critical role in pathological states, such as cancer metastasis ${ }^{54}$. CIL is the normal procedure by which a cell halts movement or alters its direction upon collision with a neighbouring cell. One important developmental process where CIL is involved is CNC migration.

Neural crest cells are multipotent progenitor cells that, subsequent to dispersing from the neuroepithelium, migrate initially in three distinct streams and further branch off into other regions, including the prospective face, branchial arches, heart and gut ${ }^{11}$. There are many factors leading CNC cells to the correct locations ${ }^{11}$. However, it is cell-cell interactions that represent a critical mainspring for $\mathrm{CNC}$ migration through establishing cell polarisation and activating the PCP signalling pathway. As CNC cells start to migrate, the cells at the front of the group experience CIL at the rear and polarise toward the open front of the collective. During this movement, there is a loosening of the space between the collective cells, allowing for polarisation towards the collective front, and can thus be responsive to the attractant signals from the surrounding tissue.

Alterations in cadherin levels including E-cadherin, $\mathrm{N}$-cadherin, cadherin-11 and protocadherins can exert substantive effects on CNC migration ${ }^{1,3,4,6,62,63}$. E-cadherin is highly expressed in the pre-migratory $\mathrm{CNC}$ cells, but E-cadherin downregulation is required for appropriate CIL behaviour during CNC cell migration ${ }^{3,11}$. Here we found that knockdown of ephrinB2 or TBC1d24 caused elevation of E-cadherin levels in $\mathrm{CNC}$ cells at the migratory stage, whereas levels of $\mathrm{N}$-cadherin remained unchanged, resulting in a reduced CIL response in CNC cells both in vitro and in vivo (Fig. $5 \mathrm{c}-\mathrm{e}$, Supplementary Fig. 3e and f). Moreover, our data support a mechanistic model for how ephrinB2 may regulate CIL in CNC cells via TBC1d24. TBC1d24 interacts with ephrinB2 through the known association of ephrinB2 and Dsh ${ }^{40-43}$, as indicated in Fig. 2. Moreover, the interaction between ephrinB2 and TBC1d24 localises TBC1d24 to the membrane, and depletion of endogenous Dsh in Xenopus embryos disrupts this localisation (Fig. 2e). The ephrinB2/Dsh/ TBC1d24 complex modulates E-cadherin levels through regulating Rab35 activity. A previous report implicated Arf6 as a substrate of $\mathrm{TBC} 1 \mathrm{~d} 24$ that regulates neuronal cell migration ${ }^{51}$. However, our results showed that, in Xenopus embryos, overexpression of Arf6 or a constitutively active form of Arf6 did not affect E-cadherin levels (Supplementary Fig. 3e) and Arf6 showed only a weak interaction with TBC1d24 (Supplementary Fig. 3a). We demonstrated that, although both Rab5 and Rab35 can robustly interact with $\mathrm{TBC} 1 \mathrm{~d} 24$ in $\mathrm{CNC}$ cells (Supplementary Fig. 3a), only inactive Rab35 induces elevated E-cadherin levels associated with inhibition of CIL (Supplementary Fig. 3e).

Eph-ephrin signalling has been implicated in regulating neural crest cell migration $22,23,25-27$, and Eph-ephrin interactions transduce signals in CNCs that can affect cell-cell repulsion or cell-cell adhesion ${ }^{60}$. These interactions result in preventing the mixing of CNC streams through contacts between CNCs and the surrounding tissue ${ }^{64}$. Our results showed that, in Xenopus CNC, the combination of the EphB4 receptor in the surrounding tissue and the ephrinB2 ligand in the CNC cells plays a role in three aspects of directed collective migration: (1) repulsion between CNC cells and the placode that are independent of TBC1d24; (2) SDF-1-directed chemotaxis of CXCR4 containing CNC cells towards the SDF-1 ligand in the placode; and (3) loss of homotypic CIL in CNC cells upon contact with the EphB-containing placode. Interestingly, the relevance of Eph-ephrin signalling in CIL has been studied in metastatic cancer cells, where it was found that homotypic collisions between two prostate cancer cells displayed CIL that was facilitated by EphA/Rho/Rho kinase signalling. Upon heterotypic collisions between prostate cancer cells and fibroblasts, the CIL response was dramatically decreased owing to enriched levels of EphB3 and EphB4 in the prostate cancer cells ${ }^{65}$.

In CNC cells, homotypic CIL among neural crest cells is postulated to restrict the protrusion generation between neighbouring cells, thus polarising the whole group for a common directional movement ${ }^{4}$. There are several steps to CIL including an initial cell-cell contact and adhesion stage $\mathrm{e}^{54}$, and this may be where ephrinB2/Dsh/TBC1d24 interactions have the most influence on CIL, through maintaining low E-cadherin levels at the cell surface in a manner independent of the EphB receptor. SDF-1 secreted from placode cells attracts neural crest cells, but placodal cells undergo CIL in response to contact with the neural crest cells, resulting in repulsion away from the neural crest cells ${ }^{62}$. Our study indicates that the homotypic CIL among ephrinB2-expressing CNC cells in conjunction with SDF-1-driven attraction towards the placode cells represents a major force promoting CNC collective migration to the placodes. Upon engagement with the EphB4-expressing placode cells, ephrinB2-expressing CNC cells suppress the chemotactic response and undergo an Eph/ephrin repulsion that is independent of TBC1d24. 
In summary, our data provide evidence that, in CNC cells of Xenopus embryos, the ephrinB2 transmembrane ligand interacts with TBC1d24, a negative regulator of Rab 35 . The scaffold protein Dsh mediates the association between ephrinB2 and TBC1d24 and leads to re-localisation of TBC1d24 to the membrane where it negatively regulates Rab 35-dependent endosome recycling of E-cadherin. This regulation maintains a low level of E-cadherin expression at the CNC cell membrane, which allows for proper CIL and migration of these cells. The interaction between the ephrinB2 ligand in the $\mathrm{CNC}$ and its cognate EphB4 receptor in the surrounding tissue induces tyrosine phosphorylation of the intracellular domain of ephrinB2, leading to disengagement of Dsh and $\mathrm{TBC} 1 \mathrm{~d} 24$, induction of repulsive activity, inhibition of the chemotactic response and ceasing the movement of these cells.

\section{Methods}

Plasmids and reagents. The cDNA clone that encodes full-length TBC1d24 was obtained from Source BioScience (GenBank ID: BC128694). The sequences of MOs are as follows; ephrinB2 MO, 5'-GAGTCCCCGCTCAGTGCCATGATCT-3'66; TBC1d24 MO, 5'- AACGGCCATATTCAGCTTCATCCAT-3'; EphB4 MO, 5'GGAGGAGCAGCTATAAATTGATCCA-3'; Dsh2 MO, 5'-TCACTTTAGTCT CCGCCATTCTGCG-3 ${ }^{\prime 42,67}$; and E-cadherin, $5^{\prime}$-AACCAGGGCCTCTTCAAC CCCATTG-3 $3^{\prime 68}$. Various HA-tagged mutants of ephrinB2 $(\Delta 4, \Delta 6, \Delta 10, \Delta 16, \Delta 19$ and Y5F) and the Dsh2 deletion mutants have been reported ${ }^{42,66}$. V5-tagged deletion mutants of TBC1d $24(\Delta \mathrm{N}, \Delta \mathrm{C}, \Delta \mathrm{TLD}, \Delta \mathrm{TBC}$ and $\Delta \mathrm{A}-\Delta \mathrm{F})$ were generated using the QuikChange II Site-Directed Mutagenesis Kit. To test whether tyrosine phosphorylation affects the ephrinB2-TBC1d24 interaction, FGFR1 KE (FGFR1 K562E), FGFR1 KD (FGFR1 C289R/K420A) and EphB4 $\Delta$ C-Flag (amino acids 1-596) were generated in the pCS2+ vector ${ }^{40}$. Clip-tag was synthesised and amplified by PCR. The Cadherin prodomain (amino acids 30-150) was replaced by an amplified Clip-tag in the pCS2-E-cadherin clone.

Embryonic injections. We obtained Xenopus embryos using standard methods ${ }^{69}$. Using the SP6 mMessage mMachine Kit (Ambion), we produced capped mRNAs that were microinjected into embryos at the indicated dose, as described in the figure legends. For the rescue of MO effects, MO-resistant mRNA was synthesised. In the case of TBC1d24-MOR, eight nucleotide substitutions were generated in wobble codons subsequent to the ATG start codon (Supplementary Fig. 2a). MOs and mRNAs were microinjected into the animal pole region in one-cell-stage embryos or the D.1.2 blastomere at the 16-cell stage as indicated in Supplementary Fig. 2c. All animal studies were approved by the NCI at Frederick Animal Care and Use Committee [ACUC].

IP and western blot analysis. Xenopus embryo lysates or LS174T cell (ATCC) lysates were prepared with ice-cold TNSG buffer $(20 \mathrm{mM}$ Tris- $\mathrm{HCl} \mathrm{pH} \mathrm{7.5,137}$ $\mathrm{mM} \mathrm{NaCl}$ and $1 \% \mathrm{NP}-40$ ). IPs were performed for $8 \mathrm{~h}$ with 25 embryo equivalent extracts using monoclonal Anti-Flag, HA or V5-agarose (Sigma-Aldrich). Endogenous protein IPs were conducted for $8 \mathrm{~h}$ with LS174T cell extracts using an antiephrinB2 antibody (1:500, C-18, Santa Cruz Biotechnology) or anti-Dsh2 antibodies (1:500, 3216, Cell Signaling). Western blot analysis was performed using anti-Flag-horseradish peroxidase (HRP)-conjugated (1:5000, A8592, Sigma), antiHA-HRP-conjugated (1:5000, 12013819001, Roche), anti-phosphotyrosine-HRPconjugated (1:1000, 4G10, Upstate Biotechnology), anti-ephrinB2 (1:1000, SAB4300456, sigma), and anti-ERK2 (1:1000, Santa Cruz Biotechnology) antibodies.

Immunofluorescence microscopy. Animal cap explants were collected at stage 10.5 and immunofluorescence microscopy was carried out using standard protocols ${ }^{42,66}$. The following primary antibodies were used: anti-V5 (1:500, G189, ABM), anti-HA (1:1000, C29F4, Cell Signaling Technology), anti-E-cadherin (1:10, $5 \mathrm{D} 3, \mathrm{DSHB})$ and anti-N-cadherin (1:20, MNCD2, DSHB)

In vitro neural crest cell migration assay. Xenopus $\mathrm{CNC}$ cells were labelled with GFD that was injected into the D.1.2 blastomere at the 16-cell stage. For single-cell collision assays, CNC cells were briefly dissociated in $\mathrm{Ca}^{2+} / \mathrm{Mg}^{2+}$-free Danilchick medium $^{3}$ and then cultured on fibronectin-coated plates $(100 \mu \mathrm{g} / \mathrm{ml})$ in Modified Danilchick Medium (MDM, NaCl $53 \mathrm{mM}, \mathrm{Na}_{2} \mathrm{CO}_{3} 11.7 \mathrm{mM}$, K Gluconate 4.27 $\mathrm{mM}, \mathrm{MgSO}_{4} 2.035 \mathrm{mM}, \mathrm{CaCl}_{2} 1.32 \mathrm{mM}$, bovine serum albumin $0.1 \%$, adjust $\mathrm{pH}$ to 8.3 with $1 \mathrm{M}$ Bicine $)^{70}$. Time-lapse images were taken using confocal microscopy. Control-Fc and EphB4-Fc (R\&D Systems) were clustered using human immunoglobulin 63 .

Co-localisation of GFP-Rab35 and Clip-E-cadherin. MOs and RNAs were injected with RNAs of GFP-Rab35 (50 pg) and Clip-E-cadherin $(25 \mathrm{pg})$ into the
D.1.2 blastomere at the 16-cell stage. GFP-positive neural crest tissues were dissected from stage 20 embryos and incubated with Clip-tag substrate (Non-cellpermeable, Clip-Surface $^{m} 547$ ) for $1 \mathrm{~h}$ at room temperature in $1 \times$ MBS. After brief washing with MDM, the neural crest tissue explants were placed on fibronectincoated plates in MDM and incubated for $30 \mathrm{~min}$ to allow the explants to attach to the plates. Time-lapse images were taken using confocal microscopy (Zeiss LSM710/780).

Whole-mount in situ hybridisation. Xenopus embryos were collected at stage 16 and $26^{69}$ for hybridisation with the Twist, slug and myoD probes. Embryos were injected with Dextran alexa-488 and various mRNAs or MOs to distinguish the injected side of embryos. The embryos were then processed for WISH using standard methods ${ }^{71}$.

TBC1d24 knockout using CRISPR/CAS9. For guide RNA design, the design too CRISPRdirect (https://crispr.dbcls.jp) application was used to scan the genome sequence for suitable CAS9 target sequences including a PAM site. As shown in Supplementary Fig. 2g, a sequence spanning 379-401 in the first exon of the TBC1d24 (5'- TGCCGTACTGTGACCCCCGA-3') on the reverse strand was selected since no off-target effects were predicted. Single-guide RNA (sgRNA) template construction, in vitro transcription of sgRNA, microinjection and genotyping were performed as described in Nakayama et al. $(2014)^{72}$. Small sections of the tail $(2 \mathrm{~mm})$ were dissected from F0 embryos and then analysed to verify mutagenesis by the direct sequencing of PCR amplicons assay ${ }^{73}$. Anterior parts of F0 embryos were used for WISH to assess CNC cell migration.

Delaunay triangulation. Individual cells were selected and used to build triangles between the neighbouring cells using the Delaunay triangulation application of Matlab software. Then each triangular shaped area was calculated. An average triangular area was normalised by the mean of the Control-Fc-treated batch of each experiment.

Invasion assay and chemoattraction assay. MOs and RNAs were injected along with GFD into the D.1.2 blastomere at the 16-cell stage, and neural crest tissue explants from GFD embryos were excised at stage 20. Neural crest tissue explants or placode tissue explants from the embryos injected with RFD were excised and placed next to the GFD-containing explants on fibronectin-coated plates in MDM and observed. After a $12 \mathrm{~h}$ incubation, the explants of the experimental group invaded (due to loss of CIL) into the normal RFD explants as demonstrated by the increased area of overlap between the explants (yellow area, white arrows). For the chemoattraction assay, GFD-injected neural crest explants and RFD-injected placode explants were dissected at stage 20 and placed about $400 \mu \mathrm{m}$ apart and incubated in low $\mathrm{Ca}^{2+} / \mathrm{Mg}^{2+} \mathrm{MDM}^{62}$. Control-Fc and EphB4-Fc (R\&D Systems) were clustered using human immunoglobulin ${ }^{74}$.

Statistical analysis. Sample size was determined as indicated in the figures and specific statistical method was not used. Dead cells and embryos were excluded from all experimental analysis. Embryos which have mis-targeted injection also were excluded (the target injection was confirmed by co-injection of lineage tracer (GFP RNAs, GFD or RFD). All experiments were performed blinded with order of testing randomised. ImageJ program was used for all quantification. All experiments were performed for at least three independent times. Normality of data was tested using Kolmogorov-Smirnov's test, D'Agostino and Pearson omnibus normality test and Shapiro-Wilk normality test using Prism5. The data were considered normal if found as normal by all three tests. Data sets following a normal distribution were compared with Student's $t$ test (two-tailed, unequal variances) or a one-way analysis of variance (ANOVA) with Dunnett's multiple comparisons post-test in Prism5. The data that did not follow a normal distribution were compared using Mann-Whitney's test or a non-parametric ANOVA (Kruskal-Wallis with Dunn's multiple comparisons post-test) using Prism5. Crosscomparisons were performed only if the overall $P$ value of the ANOVA was $<0.05$.; error bars: s.d.

Data Availability. The data that support the findings of this study and the custom code for the Delaunay triangulation application of Matlab software are available from the corresponding author upon reasonable request.

Received: 5 September 2017 Accepted: 2 August 2018 Published online: 28 August 2018

\section{References}

1. Cousin, H. Cadherins function during the collective cell migration of Xenopus cranial neural crest cells: revisiting the role of E-cadherin. Mech. Dev. 148, 79-88 (2017) 
2. Borchers, A., David, R. \& Wedlich, D. Xenopus cadherin-11 restrains cranial neural crest migration and influences neural crest specification. Development 128, 3049-3060 (2001)

3. Scarpa, E. et al. Cadherin switch during EMT in neural crest cells leads to contact inhibition of locomotion via repolarization of forces. Dev. Cell 34, 421-434 (2015).

4. Theveneau, E. et al. Collective chemotaxis requires contact-dependent cell polarity. Dev. Cell 19, 39-53 (2010).

5. Abbruzzese, G., Becker, S. F., Kashef, J. \& Alfandari, D. ADAM13 cleavage of cadherin-11 promotes CNC migration independently of the homophilic binding site. Dev. Biol. 415, 383-390 (2016).

6. Becker, S. F., Mayor, R. \& Kashef, J. Cadherin-11 mediates contact inhibition of locomotion during Xenopus neural crest cell migration. PLoS ONE 8 , e85717 (2013)

7. Kashef, J. et al. Cadherin-11 regulates protrusive activity in Xenopus cranial neural crest cells upstream of Trio and the small GTPases. Genes Dev. 23, 1393-1398 (2009).

8. Langhe, R. P. et al. Cadherin-11 localizes to focal adhesions and promotes cellsubstrate adhesion. Nat. Commun. 7, 10909 (2016).

9. Bahm, I. et al. PDGF controls contact inhibition of locomotion by regulating $\mathrm{N}$-cadherin during neural crest migration. Development 144, 2456-2468 (2017).

10. Carmona-Fontaine, C. et al. Complement fragment C3a controls mutual cell attraction during collective cell migration. Dev. Cell 21, 1026-1037 (2011).

11. Theveneau, E. \& Mayor, R. Neural crest migration: interplay between chemorepellents, chemoattractants, contact inhibition, epithelialmesenchymal transition, and collective cell migration. Wiley Interdiscip. Rev. Dev. Biol. 1, 435-445 (2012).

12. McLennan, R., Teddy, J. M., Kasemeier-Kulesa, J. C., Romine, M. H. \& Kulesa, P. M. Vascular endothelial growth factor (VEGF) regulates cranial neural crest migration in vivo. Dev. Biol. 339, 114-125 (2010).

13. Olesnicky Killian, E. C., Birkholz, D. A. \& Artinger, K. B. A role for chemokine signaling in neural crest cell migration and craniofacial development. Dev. Biol. 333, 161-172 (2009).

14. Young, H. M., Anderson, R. B. \& Anderson, C. R. Guidance cues involved in the development of the peripheral autonomic nervous system. Auton. Neurosci. 112, 1-14 (2004).

15. Sato, A. et al. FGF8 signaling is chemotactic for cardiac neural crest cells. Dev. Biol. 354, 18-30 (2011).

16. Yu, H. H. \& Moens, C. B. Semaphorin signaling guides cranial neural crest cell migration in zebrafish. Dev. Biol. 280, 373-385 (2005).

17. Gammill, L. S., Gonzalez, C. \& Bronner-Fraser, M. Neuropilin 2/semaphorin $3 \mathrm{~F}$ signaling is essential for cranial neural crest migration and trigeminal ganglion condensation. Dev. Neurobiol. 67, 47-56 (2007).

18. De Calisto, J., Araya, C., Marchant, L., Riaz, C. F. \& Mayor, R. Essential role of non-canonical Wnt signalling in neural crest migration. Development 132, 2587-2597 (2005)

19. Matthews, H. K. et al. Directional migration of neural crest cells in vivo is regulated by Syndecan-4/Rac1 and non-canonical Wnt signaling/RhoA. Development 135, 1771-1780 (2008).

20. Barriga, E. H., Franze, K., Charras, G. \& Mayor, R. Tissue stiffening coordinates morphogenesis by triggering collective cell migration in vivo. Nature 554, 523-527 (2018).

21. Huang, C., Kratzer, M. C., Wedlich, D. \& Kashef, J. E-cadherin is required for cranial neural crest migration in Xenopus laevis. Dev. Biol. 411, 159-171 (2016).

22. Smith, A., Robinson, V., Patel, K. \& Wilkinson, D. G. The EphA4 and EphB1 receptor tyrosine kinases and ephrin-B2 ligand regulate targeted migration of branchial neural crest cells. Curr. Biol. 7, 561-570 (1997).

23. Krull, C. E. et al. Interactions of Eph-related receptors and ligands confer rostrocaudal pattern to trunk neural crest migration. Curr. Biol. 7, 571-580 (1997).

24. Adams, R. H. et al. The cytoplasmic domain of the ligand ephrinB2 is required for vascular morphogenesis but not cranial neural crest migration. Cell 104, 57-69 (2001).

25. Santiago, A. \& Erickson, C. A. Ephrin-B ligands play a dual role in the control of neural crest cell migration. Development 129, 3621-3632 (2002).

26. Davy, A., Aubin, J. \& Soriano, P. Ephrin-B1 forward and reverse signaling are required during mouse development. Genes Dev. 18, 572-583 (2004).

27. MellottD. O. \& BurkeR. D. Divergent roles for Eph and Ephrin in avian cranial neural crest. BMC. Dev. Biol. 8, 56 (2008).

28. Wei, S. et al. ADAM13 induces cranial neural crest by cleaving class B Ephrins and regulating Wnt signaling. Dev. Cell 19, 345-352 (2010).

29. Noh, H., Park, E. \& Park, S. In vivo expression of ephrinA5-Fc in mice results in cephalic neural crest agenesis and craniofacial abnormalities. Mol. Cells 37, 59-65 (2014).

30. Kuriyama, S. \& Mayor, R. Molecular analysis of neural crest migration. Philos. Trans. R. Soc. Lond. B. Biol. Sci. 363, 1349-1362 (2008).
31. Allaire, P. D. et al. Interplay between Rab35 and Arf6 controls cargo recycling to coordinate cell adhesion and migration. J. Cell Sci. 126, 722-731 (2013).

32. Falace, A. et al. TBC1D24, an ARF6-interacting protein, is mutated in familial infantile myoclonic epilepsy. Am. J. Hum. Genet. 87, 365-370 (2010).

33. Campeau, P. M. et al. The genetic basis of DOORS syndrome: an exomesequencing study. Lancet Neurol. 13, 44-58 (2014).

34. Guven, A. \& Tolun, A. TBC1D24 truncating mutation resulting in severe neurodegeneration. J. Med. Genet. 50, 199-202 (2013).

35. Bush, J. O. \& Soriano, P. Eph/ephrin signaling: genetic, phosphoproteomic, and transcriptomic approaches. Semin. Cell Dev. Biol. 23, 26-34 (2012).

36. Daar, I. O. Non-SH2/PDZ reverse signaling by ephrins. Semin. Cell Dev. Biol. 23, 65-74 (2012).

37. Cho, H. J. et al. EphrinB1 interacts with $\mathrm{CNK} 1$ and promotes cell migration through c-Jun N-terminal kinase (JNK) activation. J. Biol. Chem. 289, 18556-18568 (2014)

38. Hwang, Y. S. \& Daar, I. O. A frog's view of EphrinB signaling. Genesis 55, https://doi.org/10.1002/dvg.23002 (2017).

39. Shnitsar, I. \& Borchers, A. PTK7 recruits dsh to regulate neural crest migration. Development 135, 4015-4024 (2008).

40. Lee, H. S. et al. Fibroblast growth factor receptor-induced phosphorylation of EphrinB1 modulates its interaction with Dishevelled. Mol. Biol. Cell 20, 124-133 (2009).

41. Tanaka, M., Kamo, T., Ota, S. \& Sugimura, H. Association of Dishevelled with Eph tyrosine kinase receptor and ephrin mediates cell repulsion. EMBO J 22, 847-858 (2003).

42. Lee, H. S. et al. Dishevelled mediates ephrinB1 signalling in the eye field through the planar cell polarity pathway. Nat. Cell Biol. 8, 55-63 (2006).

43. Mao, Y., Huang, X., Zhao, J. \& Gu, Z. Preliminary identification of potential PDZ-domain proteins downstream of ephrin B2 during osteoclast differentiation of RAW264.7 cells. Int. J. Mol. Med. 27, 669-677 (2011).

44. Holland, S. J. et al. Juxtamembrane tyrosine residues couple the Eph family receptor EphB2/Nuk to specific SH2 domain proteins in neuronal cells. $E M B O$ J 16, 3877-3888 (1997).

45. Bruckner, K., Pasquale, E. B. \& Klein, R. Tyrosine phosphorylation of transmembrane ligands for Eph receptors. Science 275, 1640-1643 (1997).

46. Tanaka, M., Kamata, R. \& Sakai, R. Phosphorylation of ephrin-B1 via the interaction with claudin following cell-cell contact formation. EMBO J $\mathbf{2 4}$ 3700-3711 (2005)

47. Chong, L. D., Park, E. K., Latimer, E., Friesel, R. \& Daar, I. O. Fibroblast growth factor receptor-mediated rescue of $\mathrm{x}$-ephrin $\mathrm{B} 1$-induced cell dissociation in Xenopus embryos. Mol. Cell. Biol. 20, 724-734 (2000).

48. Davy, A. \& Soriano, P. Ephrin-B2 forward signaling regulates somite patterning and neural crest cell development. Dev. Biol. 304, 182-193 (2007).

49. Milet, C. \& Monsoro-Burq, A. H. Dissection of Xenopus laevis neural crest for in vitro explant culture or in vivo transplantation. J. Vis. Exp. https://doi.org/ $10.3791 / 51118$ (2014).

50. Uytterhoeven, V., Kuenen, S., Kasprowicz, J., Miskiewicz, K. \& Verstreken, P. Loss of skywalker reveals synaptic endosomes as sorting stations for synaptic vesicle proteins. Cell 145, 117-132 (2011).

51. Falace, A. et al. TBC1D24 regulates neuronal migration and maturation through modulation of the ARF6-dependent pathway. Proc. Natl Acad. Sci. USA 111, 2337-2342 (2014)

52. Desclozeaux, M. et al. Active Rab11 and functional recycling endosome are required for E-cadherin trafficking and lumen formation during epithelial morphogenesis. Am. J. Physiol. Cell Physiol. 295, C545-C556 (2008).

53. Palacios, F., Tushir, J. S., Fujita, Y. \& D'Souza-Schorey, C. Lysosomal targeting of E-cadherin: a unique mechanism for the down-regulation of cell-cell adhesion during epithelial to mesenchymal transitions. Mol. Cell. Biol. 25, 389-402 (2005).

54. Stramer, B. \& Mayor, R. Mechanisms and in vivo functions of contact inhibition of locomotion. Nat. Rev. Mol. Cell Biol. 18, 43-55 (2017).

55. Charrasse, S., Comunale, F., De Rossi, S., Echard, A. \& Gauthier-Rouviere, C. Rab35 regulates cadherin-mediated adherens junction formation and myoblast fusion. Mol. Biol. Cell 24, 234-245 (2013).

56. Zerial, M. \& McBride, H. Rab proteins as membrane organizers. Nat. Rev. Mol. Cell Biol. 2, 107-117 (2001).

57. Coulthard, M. G. et al. Eph/Ephrin signaling in injury and inflammation. Am. J. Pathol. 181, 1493-1503 (2012).

58. Arboleda-Estudillo, Y. et al. Movement directionality in collective migration of germ layer progenitors. Curr. Biol. 20, 161-169 (2010).

59. Li, L. et al. E-cadherin plays an essential role in collective directional migration of large epithelial sheets. Cell. Mol. Life Sci. 69, 2779-2789 (2012).

60. Zimmer, M., Palmer, A., Kohler, J. \& Klein, R. EphB-ephrinB bi-directional endocytosis terminates adhesion allowing contact mediated repulsion. Nat. Cell Biol. 5, 869-878 (2003).

61. Lu, Q., Sun, E. E., Klein, R. S. \& Flanagan, J. G. Ephrin-B reverse signaling is mediated by a novel PDZ-RGS protein and selectively inhibits $G$ proteincoupled chemoattraction. Cell 105, 69-79 (2001). 
62. Theveneau, E. et al. Chase-and-run between adjacent cell populations promotes directional collective migration. Nat. Cell Biol. 15, 763 (2013).

63. McCusker, C., Cousin, H., Neuner, R. \& Alfandari, D. Extracellular cleavage of cadherin-11 by ADAM metalloproteases is essential for Xenopus cranial neural crest cell migration. Mol. Biol. Cell 20, 78-89 (2009).

64. Theveneau, E. \& Mayor, R. Neural crest delamination and migration: from epithelium-to-mesenchyme transition to collective cell migration. Dev. Biol. 366, 34-54 (2012).

65. Astin, J. W. et al. Competition amongst Eph receptors regulates contact inhibition of locomotion and invasiveness in prostate cancer cells. Nat. Cell Biol. 12, 1194-1204 (2010).

66. Ji, Y. J. et al. EphrinB2 affects apical constriction in Xenopus embryos and is regulated by ADAM10 and flotillin-1. Nat. Commun. 5, 3516 (2014).

67. Sheldahl, L. C. et al. Dishevelled activates Ca2+ flux, PKC, and CamKII in vertebrate embryos. J. Cell Biol. 161, 769-777 (2003).

68. Nandadasa, S., Tao, Q., Menon, N. R., Heasman, J. \& Wylie, C. N- and Ecadherins in Xenopusare specifically required in the neural and non-neural ectoderm, respectively, for F-actin assembly and morphogenetic movements. Development 136, 1327-1338 (2009).

69. Moody, S. A. Cell lineage analysis in Xenopus embryos. Methods Mol. Biol. 135, 331-347 (2000).

70. Cousin, H. \& Alfandari, D. Cranial neural crest explants. Cold Spring Harb. Protoc. 2018, https://doi.org/10.1101/pdb.prot097394 (2018).

71. Harland, R. M. In situ hybridization: an improved whole-mount method for Xenopus embryos. Methods Cell Biol. 36, 685-695 (1991).

72. Nakayama, T. et al. Cas9-based genome editing in Xenopus tropicalis. Methods Enzymol. 546, 355-375 (2014).

73. Nakayama, T. et al. Simple and efficient CRISPR/Cas9-mediated targeted mutagenesis in Xenopus tropicalis. Genesis 51, 835-843 (2013).

74. Cho, H. J. et al. EphrinB1 promotes cancer cell migration and invasion through the interaction with RhoGDI1. Oncogene 37, 861-872 (2018).

\section{Acknowledgements}

We thank Giovanna Tosato, Jairaj Acharya, and Shyam Sharan for critically reviewing the manuscript. We thank Ming Zhou for performing the Mass spectrometric analysis of our ephrinB2 immunoprecipitates and Christopher Westlake for providing the Arf6, Rab5, Rab7, Rab 9 and Rab11 constructs. This research was supported by the Intramural
Research Program of the National Institutes of Health, National Cancer Institute. J.Y. is the recipient of Korean Visiting Scientist Training Award (KVSTA) fellowship, supported by Korea Health Industry Development Institute (KHIDI) in cooperation with NIH (HI3C1158).

\section{Author contributions}

J.Y. and I.D. designed the experiments, analysed the data and wrote the manuscript; J.Y., Y.H., M.L., J.S., H.C. and L.K. performed the experiments.

\section{Additional information}

Supplementary Information accompanies this paper at https://doi.org/10.1038/s41467018-05924-9.

Competing interests: The authors declare no competing interests.

Reprints and permission information is available online at http://npg.nature.com/ reprintsandpermissions/

Publisher's note: Springer Nature remains neutral with regard to jurisdictional claims in published maps and institutional affiliations.

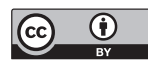

Open Access This article is licensed under a Creative Commons Attribution 4.0 International License, which permits use, sharing, adaptation, distribution and reproduction in any medium or format, as long as you give appropriate credit to the original author(s) and the source, provide a link to the Creative Commons license, and indicate if changes were made. The images or other third party material in this article are included in the article's Creative Commons license, unless indicated otherwise in a credit line to the material. If material is not included in the article's Creative Commons license and your intended use is not permitted by statutory regulation or exceeds the permitted use, you will need to obtain permission directly from the copyright holder. To view a copy of this license, visit http://creativecommons.org/ licenses/by/4.0/.

(C) The Author(s) 2018 